\title{
A Multidomain Discretization of the Richards Equation in Layered Soil
}

\author{
Heiko Berninger, Ralf Kornhuber, and \\ Oliver Sander
}

Bericht Nr. 358

März 2013

Key words: Richards equation, domain decomposition, layered soil, nonlinear discretization, robustness, surface water

AMS Subject Classifications: 65N30, 65N55, 76S05

Institut für Geometrie und Praktische Mathematik RWTH Aachen

Templergraben 55, D-52056 Aachen (Germany)

This work was supported by the BMBF-Programm ,,Mathematik für Innovationen in Industrie und Dienstleistungen “. 


\title{
A MULTIDOMAIN DISCRETIZATION OF THE RICHARDS EQUATION IN LAYERED SOIL
}

\author{
HEIKO BERNINGER, RALF KORNHUBER, AND OLIVER SANDER
}

\begin{abstract}
We consider the Richards equation on a domain that is decomposed into nonoverlapping layers, i.e., the decomposition has no cross points. We assume that the saturation and permeability functions are space-independent on each subdomain. Kirchhoff transformation of each subdomain problem separately then leads to a set of semi-linear equations, which can each be solved efficiently using monotone multigrid. The transformed subdomain problems are coupled by nonlinear continuity and flux conditions. This nonlinear coupled problem can be solved using substructuring methods like the Dirichlet-Neumann or Robin iteration. We give several numerical examples showing the discretization error, the solver robustness under variations of the soil parameters and a hydrological example with four soil layers and surface water.
\end{abstract}

\section{INTRODUCTION}

The Richards equation [7, 20, 33, is a well-established model for saturatedunsaturated groundwater flow. In its most general form it can be written as

$$
n(x) \theta(x, p)_{t}+\operatorname{div} \mathbf{v}(x, p)=f, \quad \mathbf{v}(x, p)=-K_{h}(x) k r(x, \theta(x, p)) \nabla(p-z)
$$

for $(x, t) \in \Omega \times(0, T)$. The first equation represents mass conservation with a source term $f$, while the second one is a generalized Darcy law 21] for the water flux $\mathbf{v}$. The flux $\mathbf{v}$ includes a gravity term $\nabla z$, where $z$ is the third component of $x$, and the $z$-axis points downwards. The dependencies on $x \in \Omega \subset \mathbb{R}^{3}$ indicate the heterogeneities of the soil. The parameters $n: \Omega \rightarrow(0,1)$ and $K_{h}: \Omega \rightarrow \mathbb{R}^{+}$are the porosity and the hydraulic conductivity, respectively. The behaviour of the porous medium is further captured by the soil water retention relationship $\theta(x, \cdot): \mathbb{R} \rightarrow$ $\left[\theta_{m}(x), \theta_{M}(x)\right]$, and the relative permeability $k r(x, \cdot):\left[\theta_{m}(x), \theta_{M}(x)\right] \rightarrow[0,1]$. For any $x \in \Omega$, the saturation $\theta$ of the soil stays between the residual and the maximal saturation $\theta_{m}(x), \theta_{M}(x) \in[0,1]$. Furthermore, it is an increasing function of the water pressure $p$ (in case of $p>0$ ) or the capillary pressure (in case of $p \leq 0$ ) for any fixed $x \in \Omega$, while, again for fixed $x$, the function $\theta \mapsto k r(x, \theta)$ is increasing with respect to $\theta$. The unit of the physical pressure $p$ is given in meters (of a water column).

We use concrete representations according to Brooks and Corey [18] of the parameter functions $\theta(x, \cdot)$ and $k r(x, \cdot)$ given in terms of two soil parameters which depend on $x$ : the bubbling pressure $p_{b}(x)<0$ and the pore size distribution factor $\lambda(x)>0$. By the property $k r(x, \cdot)>0$ with $k r(x, \theta(p)) \rightarrow 0$ for $p \rightarrow-\infty$, these functions induce a degeneracy in the elliptic-parabolic equation (1). Moreover,

1991 Mathematics Subject Classification. MSC (2000): 65N30, 65N55, 76 S05.

This work was supported by the BMBF-Programm "Mathematik für Innovationen in Industrie und Dienstleistungen". 
seepage phenomena lead to nonlinear nonsmooth outflow boundary conditions of Signorini's type at the interface between soil and air. These features leads to difficulties both in the analytical and numerical treatment of this equation. In fact, existence and uniqueness results are restricted to spatially homogeneous parameter functions $\theta(\cdot)$ and $k r(\cdot)[1,2,3$.

Big slopes of the parameter functions may have a negative effect on Newton's method applied to the corresponding finite dimensional algebraic system, e.g., in case of very coarse sand with small bubbling pressures. Therefore, in contrast to concentrating on the discretization first and on the algebraic solution later (as in [4, 24, 35, 37, 38,), we recently derived a Kirchhoff finite element discretization which is solver-friendly in the sense that iterative solution of the resulting spatial problems by monotone multigrid methods is robust with respect to soil parameters and exhibits similar convergence speed as in the saturated linear case [13. However, this approach heavily relies on the reformulation of the given quasilinear spatial problems in terms of convex minimization, based on Kirchhoff transformation. Therefore, it is restricted to homogeneous soil, i.e., spatially homogeneous parameter functions $\theta(\cdot)$ and $k r(\cdot)$.

The purpose of this paper is to extend the Kirchhoff finite element approach to the heterogeneous case, i.e., to spatially varying parameter functions $\theta(x, \cdot)$ and $k r(x, \cdot)$. In hydrologically realistic cases, heterogeneous soil is often built of layers of different homogeneous soil types. Therefore, we restrict ourselves to situations in which the dependency of the parameter functions $\theta(x, \cdot)$ and $k r(x, \cdot)$ on $x \in \Omega$ is not completely arbitrary, but in which we have a nonoverlapping decomposition of $\Omega$ into subdomains $\Omega_{i}, i=1, \ldots, n$, without cross points (see [14 for possible extensions in this direction) and $x$-independent $\theta_{i}(\cdot)$ and $k r_{i}(\cdot)$ for each $\Omega_{i}$. Under this assumption, we can derive a generalized multidomain formulation of the time-discretized Richards equation (1): After Kirchhoff transformation in the subdomains $\Omega_{i}$, continuity of physical pressure and fluxes across the interfaces transforms into nonlinear transmission conditions across the interfaces. Finite element discretization and node-wise application of the inverse Kirchhoff discretization then provides a novel multidomain discretization. In each subdomain $\Omega_{i}$ it can be interpreted as a standard finite element discretization with inexact quadrature [13, or alternatively as a finite element method with a modified metric [34. Using a model problem with known exact solution, we numerically found optimal convergence rates both in the $H^{1}$ - and the $L^{2}$-norm, whereas theoretical justification is still open.

By construction, our multidomain discretization is solver-friendly in the sense that existing nonlinear domain decomposition methods [10, 11, 12] can be applied in combination with fast and robust subdomain solvers. Concerning mesh dependence, extensive numerical studies revealed surprising similarities of nonlinear versions of Dirichlet-Neumann and Robin iterations to their linear counterpart [12. Here, we concentrate on the numerical assessment of robustness with respect to soil parameters. The ill-conditioned, inverse Kirchhoff transformation enters the iteration via the interface conditions and thus, in contrast to the homogeneous case, is no longer separated from the solution process. Nevertheless, both for Dirichlet-Neumann and Robin methods, we found surprising robustness of the convergence rates with respect to the whole range of practically relevant values of pore size distribution and bubbling pressure. 
Numerical approximation of outflow boundary conditions of Signorini-type often relies on regularization [30, 36. As our approach is based on convexity rather than on smoothness, outflow boundary conditions can be easily incorporated in terms of variational inequalities. No regularization and thus no regularization parameters are needed. This is illustrated by a numerical example with real-life data taken from [8]: We simulate surface water seeping into unsaturated, layered soil and seeping out again once the soil is saturated due to subsurface flow. Surface water is described by a simple compartment model. Coupling is performed by pressure continuity and mass conservation across the interface using Signorini-type outflow boundary conditions. This additional nonsmooth nonlinearity turns out not to cause any problems concerning efficiency and reliability of the overall solution process. Extension to more complicated situations, like, e.g., flooding, is the subject of current research.

The paper is organized as follows. In Section 2 we formally state the multidomain formulation of the Richards equation both in the physical pressure $p$ and after Kirchhoff transformation. Section 3 gives discretizations both in time and space. To make this paper self-contained, Section 4 recalls substructuring algorithms for the solution of the coupled algebraic systems, concentrating on the Dirichlet-Neumann and the Robin method. Finally, Section 5 is devoted to numerical examples. First, we assess the discretization error of our multidomain discretization (Subsection 5.1). As mesh-dependence of Dirichlet-Neumann and Robin iterations has been investigated elsewhere [12, we focus on robustness with respect to variations of the soil parameters in Subsection 5.2 Finally we present the simulation of a surface / groundwater seepage process with saturated-unsaturated layered soil and realistic hydrological data (Subsection 5.3).

\section{RichaRdS EQUATION IN LAYERED SOIL}

2.1. Multidomain formulation in physical variables. Let $\Omega \subset \mathbb{R}^{d}, d=1,2,3$, be a bounded Lipschitz domain decomposed into non-overlapping subdomains $\Omega_{i}$, $i=1, \ldots, n, n \in \mathbb{N}$, which also have Lipschitz boundaries. Then the interfaces $\Gamma_{i j}:=\bar{\Omega}_{i} \cap \bar{\Omega}_{j}, 1 \leq i, j \leq n, i \neq j$, are Lipschitz continuous, too, and the normal $\mathbf{n}_{i j}$ on $\Gamma_{i j}$ in the direction of $\Omega_{j}$ exists almost everywhere. We assume that we do not have cross points, i.e., $\Gamma_{i j} \cap \Gamma_{i^{\prime} j^{\prime}}=\emptyset$ for $i \neq i^{\prime}$ or $j \neq j^{\prime}$, so that the subdomains form layers in $\Omega$ as shown in Figure 1 . ordered by $1, \ldots, n$ with $\Omega_{1}$ on top and $\Omega_{n}$ on the bottom. Therefore, only the $\Gamma_{i j}$ with $i, j=1, \ldots, n$ and $j=i \pm 1$ are nonempty.

Each layer $\Omega_{i}$ is associated to a set of soil parameters $n_{i}, K_{h, i}, \theta_{m, i}, \theta_{M, i}, p_{b, i}$ and $\lambda_{i}$, as described in the introduction. The saturation-pressure relations $\theta_{i}(\cdot)$ and the relative permeability-saturation functions $k r_{i}(\cdot)$ associated to the subdomain $\Omega_{i}$ are $x$-independent and given by the formulas

$$
\begin{aligned}
\theta_{i}(p) & = \begin{cases}\theta_{m, i}+\left(\theta_{M, i}-\theta_{m, i}\right)\left(\frac{p}{p_{b, i}}\right)^{-\lambda_{i}} & \text { for } p \leq p_{b, i} \\
\theta_{M, i} & \text { for } p \geq p_{b, i}\end{cases} \\
k r_{i}(\theta) & =\left(\frac{\theta-\theta_{m, i}}{\theta_{M, i}-\theta_{m, i}}\right)^{3+\frac{2}{\lambda_{i}}}, \quad \theta \in\left[\theta_{m, i}, \theta_{M, i}\right],
\end{aligned}
$$




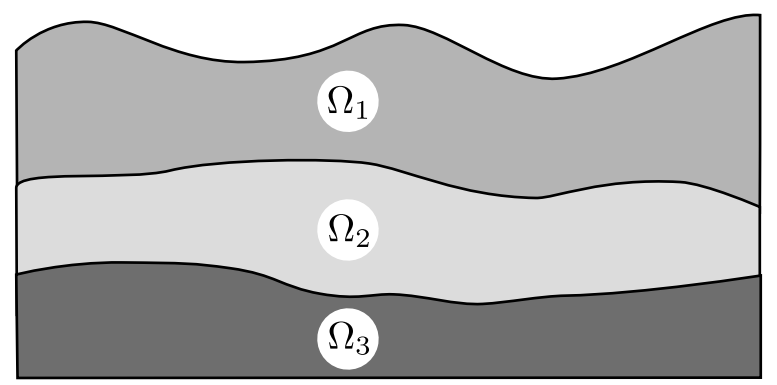

FIGURE 1. Decomposition of $\Omega$ into layers containing different soil types

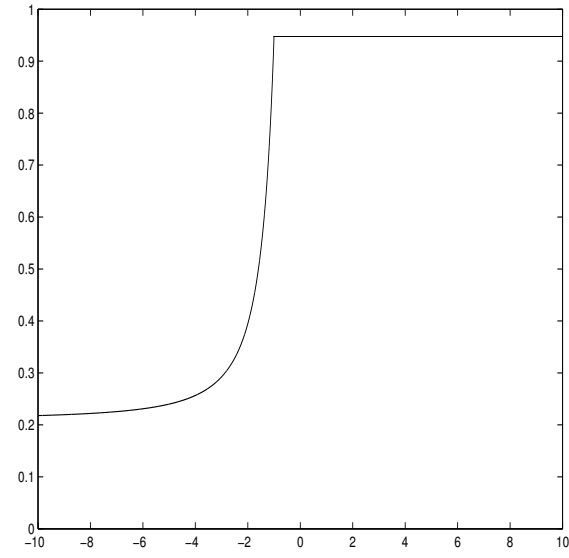

FiguRE 2. $p \mapsto \theta_{i}(p)$

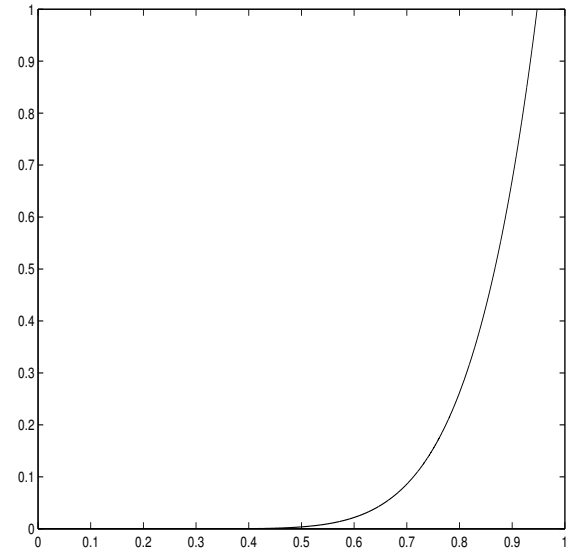

FiguRE $3 . \theta \mapsto k r_{i}(\theta)$

given by Brooks and Corey [18] and Burdine [19]. See Figures 2 and 3 for typical shapes of these functions. We call this setting layered soil from now on.

Now, we formulate our problem for saturated-unsaturated groundwater flow in this type of heterogeneous porous media: Find $\tilde{p}$ on $\Omega \times(0, T)$ satisfying suitable initial and boundary conditions to be specified below, such that with $\tilde{p}_{i}:=\tilde{p}_{\mid \Omega_{i} \times(0, T)}$ and abbreviating $\tilde{f}_{i}:=f_{\mid \Omega_{i} \times(0, T)}$ we have for all $i=1, \ldots, n$

$$
n_{i} \theta_{i}\left(\tilde{p}_{i}\right)_{t}-\operatorname{div}\left(K_{h, i} k r_{i}\left(\theta_{i}\left(\tilde{p}_{i}\right)\right) \nabla\left(\tilde{p}_{i}-z\right)\right)=f_{i} \quad \text { in } \Omega_{i} \times(0, T),
$$

and for all $i=1, \ldots, n-1, j=i+1$, the interface conditions

$$
\begin{aligned}
\tilde{p}_{i} & =\tilde{p}_{j} \\
K_{h, i} k r_{i}\left(\theta_{i}\left(\tilde{p}_{i}\right)\right) \nabla\left(\tilde{p}_{i}-z\right) \cdot \mathbf{n}_{i j} & =K_{h, j} k r_{j}\left(\theta_{j}\left(\tilde{p}_{j}\right)\right) \nabla\left(\tilde{p}_{j}-z\right) \cdot \mathbf{n}_{i j}
\end{aligned}
$$

hold on $\Gamma_{i j} \times(0, T)$. One can show that the weak form of the global Richards equation (1) is equivalent to the weak form of the multidomain problem (4)-(6). The former entails continuity of the pressure (5) as well as of the water flux, i.e., mass conservation (6), compare [10] and see also Section 3.1.2.

2.2. Boundary conditions. On Lipschitz submanifolds $\gamma_{D}$ and $\gamma_{N}$ of $\partial \Omega$ we consider Dirichlet and Neumann boundary conditions $\tilde{p}=\tilde{p}_{D}$ and $\tilde{\mathbf{v}} \cdot \mathbf{n}=f_{N}$, respectively, with admissible and compatible functions $p_{D}$ and $f_{N}$. Here, the water flux 
$\tilde{\mathbf{v}}$ is the dual variable corresponding to the primal variable $\tilde{p}$ by (1) and $\mathbf{n}$ is the outward normal on $\partial \Omega$. Robin boundary conditions of different type [10, 15] can also be included. In this paper, we only apply them on the interfaces in connection with the Robin method, see Subsection 4.2

Our formulation can easily accommodate another important nontrivial boundary condition. On the interface between a porous medium and air, one can sometimes observe seepage faces. There, the water pressure cannot exceed zero, water can flow out of the porous medium only if the water pressure is zero and there is no flow otherwise. Mathematically, such a behaviour on a Lipschitz submanifold $\gamma_{S}$ of $\partial \Omega$ can be described by the Signorini-type condition [10, 13, 36]

$$
\tilde{p} \leq 0, \quad \tilde{\mathbf{v}} \cdot \mathbf{n} \geq 0, \quad \tilde{p} \cdot(\tilde{\mathbf{v}} \cdot \mathbf{n})=0 \quad \text { a.e. on } \gamma_{S} \times(0, T) .
$$

A priori, it is unknown where outflow and where noflow occurs, i.e., we actually have a free boundary value problem. We do not allow nonempty intersections $\bar{\gamma}_{S} \cap \Gamma_{i j}$, because a substructuring formulation is lacking a rigorous justification in this case [10].

2.3. Multidomain formulation in generalized variables via local Kirchhoff transformation. We now turn the quasilinear equation (4) on each subdomain into a corresponding semi-linear equation as follows. To each of the subdomain problems (4) we apply a Kirchhoff transformation (see, e.g., [3, 23]), defined by

$$
\kappa_{i}: \tilde{p}_{i} \mapsto \tilde{u}_{i}:=\int_{0}^{\tilde{p}_{i}} k r_{i}\left(\theta_{i}(q)\right) d q
$$

where the new variable $\tilde{u}_{i}$ is called generalized pressure. The chain rule provides

$$
\nabla \tilde{u}_{i}=\kappa_{i}^{\prime}\left(\tilde{p}_{i}\right) \nabla \tilde{p}_{i}=k r_{i}\left(\theta_{i}\left(\tilde{p}_{i}\right)\right) \nabla \tilde{p}_{i}
$$

and the saturation as a function of $\tilde{u}_{i}$ shall be denoted by

$$
M_{i}\left(\tilde{u}_{i}\right):=\theta_{i}\left(\kappa_{i}^{-1}\left(\tilde{u}_{i}\right)\right) .
$$

Figures 4 and 5 show the typical behaviour of the inverse transformation $\kappa_{i}^{-1}$ and of $M_{i}$. Note that by superposition, the real functions $\kappa_{i}$ and $\kappa_{i}^{-1}$ can also be regarded as superposition operators acting on spaces of real-valued functions. Denoting the unit vector in the direction of gravity by $e_{z}:=\nabla z$, the transformed subdomain problems and interface conditions (4)-(6) read

$$
n_{i} M_{i}\left(\tilde{u}_{i}\right)_{t}-\operatorname{div}\left(K_{h, i}\left(\nabla \tilde{u}_{i}-k r_{i}\left(M_{i}\left(\tilde{u}_{i}\right)\right) e_{z}\right)\right)=\tilde{f}_{i} \quad \text { in } \Omega_{i} \times(0, T)
$$

for $i=1, \ldots, n$, and for all $i=1, \ldots, n-1, j=i+1$, the interface conditions

$$
\begin{aligned}
\kappa_{i}^{-1} \tilde{u}_{i} & =\kappa_{j}^{-1} \tilde{u}_{j} \\
K_{h, i}\left(\nabla \tilde{u}_{i}-k r_{i}\left(M_{i}\left(\tilde{u}_{i}\right)\right) e_{z}\right) \cdot \mathbf{n}_{i j} & =K_{h, j}\left(\nabla \tilde{u}_{j}-k r_{j}\left(M_{j}\left(\tilde{u}_{j}\right)\right) e_{z}\right) \cdot \mathbf{n}_{i j}
\end{aligned}
$$

hold on $\Gamma_{i j} \times(0, T)$. The advantage of the Kirchhoff transformation for the homogeneous cases is that the subproblems (9) are semilinear equations whereas the untransformed problems (4) are quasilinear. Since $\kappa_{i}(0)=0$ for all $i=1, \ldots, n$ and the water flux is

$$
\tilde{\mathbf{v}}=-K_{h, i}\left(\nabla \tilde{u}_{i}-k r_{i}\left(M_{i}\left(\tilde{u}_{i}\right)\right) e_{z}\right)
$$

in the transformed variable $\tilde{u}$ on $\Omega \times(0, T)$ given by $\tilde{u}_{\mid \Omega_{i} \times(0, T)}:=\tilde{u}_{i}$ for $i=1, \ldots, n$, the transformed Signorini-type boundary condition reads

$$
\tilde{u} \leq 0, \quad \tilde{\mathbf{v}} \cdot \mathbf{n} \geq 0, \quad \tilde{u} \cdot(\tilde{\mathbf{v}} \cdot \mathbf{n})=0 \quad \text { a.e. on } \gamma_{S} \times(0, T) .
$$




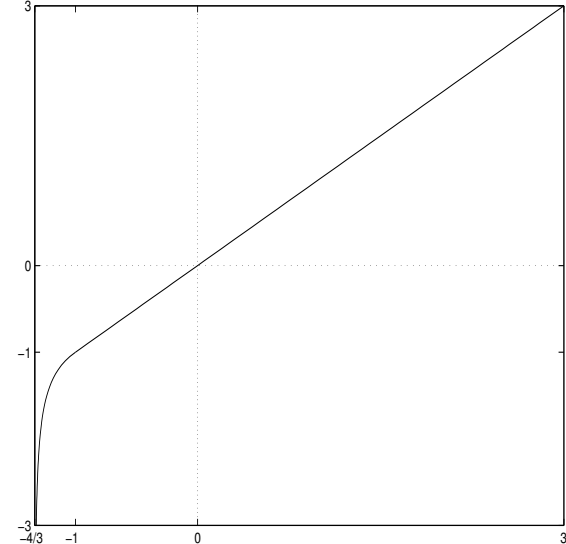

FiguRE 4. $u \mapsto \kappa_{i}^{-1}(u)$

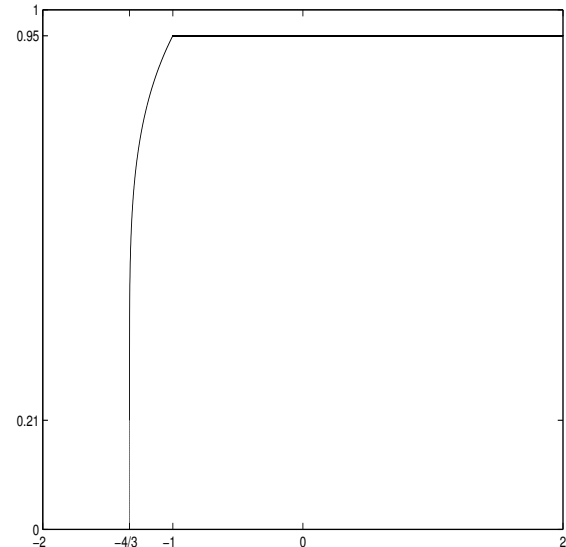

FiguRE 5. $u \mapsto M_{i}(u)$

Dirichlet and Neumann boundary conditions are transformed accordingly. In particular, transformed homogeneous boundary conditions are again homogeneous. We refer to [9] and [8, Sec. 1.5.4] for a detailed discussion of the conditions needed to prove equivalence of untransformed and transformed problems.

\section{Multidomain Discretization for the Richards EQUation}

\subsection{Time-discrete physical and generalized multidomain formulation.}

3.1.1. Strong form. Let $0=t_{0}<t_{1}<\ldots<t_{N}=T$ be a partition of $[0, T]$, $k$ the time step number, and $\tau_{k}:=t_{k}-t_{k-1}$ for $k \in\{1, \ldots, N\}$ the time step size. Let $\bar{p}_{i}$ be the discrete solution on the $i$-th subdomain for the time $t_{k-1}$. We discretize (4) implicitly in the main part of the spatial derivative and explicitly in the convective, i.e., the gravitational part. Therefore, at time $t_{k}$ the time-discrete spatial multidomain problem in physical variables is to find $p_{i}$ on $\Omega_{i}$ such that

$$
n_{i} \theta_{i}\left(p_{i}\right)-\tau_{k} \operatorname{div}\left(K_{h, i} k r_{i}\left(\theta_{i}\left(p_{i}\right)\right) \nabla p_{i}\right)=f_{i} \quad \text { in } \Omega_{i}
$$

holds for $i=1, \ldots, n$ with coupling conditions

$$
p_{i}=p_{j}
$$

$$
\tau_{k} K_{h, i} k r_{i}\left(\theta_{i}\left(p_{i}\right)\right) \nabla p_{i} \cdot \mathbf{n}_{i j}-f_{i j}=\tau_{k} K_{h, j} k r_{j}\left(\theta_{j}\left(p_{j}\right)\right) \nabla p_{j} \cdot \mathbf{n}_{i j}-f_{j i} \quad \text { on } \Gamma_{i j}
$$

for $i=1, \ldots, n-1, j=i+1$. Setting $\bar{f}_{i}(\cdot):=\tilde{f}_{i}\left(\cdot, t_{k}\right)$ in $\Omega_{i}$, we abbreviate

$$
f_{i}:=\tau_{k} \bar{f}_{i}+n_{i} \theta_{i}\left(\bar{p}_{i}\right)-\tau_{k} K_{h, i} k r_{i}\left(\theta_{i}\left(\bar{p}_{i}\right)\right)_{z}
$$

as well as

$$
f_{i j}:=\tau_{k} K_{h, i} k r_{i}\left(\theta_{i}\left(\bar{p}_{i}\right)\right) e_{z} \cdot \mathbf{n}_{i j} \quad \text { and } \quad f_{j i}:=\tau_{k} K_{h, j} k r_{j}\left(\theta_{j}\left(\bar{p}_{j}\right)\right) e_{z} \cdot \mathbf{n}_{i j} .
$$

By $u_{i}:=\kappa_{i}\left(p_{i}\right), \bar{u}_{i}:=\kappa_{i}\left(\bar{p}_{i}\right)$ and replacing $\theta_{i}\left(p_{i}\right)$ and $\theta_{i}\left(\bar{p}_{i}\right)$ by $M_{i}\left(u_{i}\right)$ and $M_{i}\left(\bar{u}_{i}\right)$, respectively, in these formulas with the same set of indices $i$ and $j$, the corresponding time-discrete version of the Kirchhoff-transformed multidomain formulation (9)(11) reads

$$
n_{i} M_{i}\left(u_{i}\right)-\tau_{k} \operatorname{div}\left(K_{h, i} \nabla u_{i}\right)=f_{i} \quad \text { in } \Omega_{i}
$$


with coupling conditions

$$
\begin{aligned}
\kappa_{i}^{-1} u_{i} & =\kappa_{j}^{-1} u_{j} & & \text { on } \Gamma_{i j} \\
\tau_{k} K_{h, i} \nabla u_{i} \cdot \mathbf{n}_{i j}-f_{i j} & =\tau_{k} K_{h, j} \nabla u_{j} \cdot \mathbf{n}_{i j}-f_{j i} & & \text { on } \Gamma_{i j} .
\end{aligned}
$$

The implicit-explicit time discretization of the water flux 12 at the time $t_{k}$ reads

$$
\mathbf{v}=-K_{h, i}\left(\nabla u_{i}-k r_{i}\left(M_{i}\left(\bar{u}_{i}\right)\right) e_{z}\right) \quad \text { in } \Omega_{i}
$$

so that the time-discrete Signorini-type boundary condition $(13)$ can be written as

$$
u \leq 0, \quad \mathbf{v} \cdot \mathbf{n} \geq 0, \quad u \cdot(\mathbf{v} \cdot \mathbf{n})=0 \quad \text { on } \gamma_{S},
$$

where $u$ is defined by $u:=u_{i}$ in $\Omega_{i}$.

3.1.2. Weak form. We now turn to the weak formulation of $\sqrt{14}-(16)$ and its Kirchhoff-transformed variant (18)-(20). For simplicity and without loss of generality we restrict ourselves to homogeneous Dirichlet boundary conditions on $\partial \Omega$ in this and in the next section. We use the Sobolev spaces $H^{1}\left(\Omega_{i}\right)$ and $H_{0}^{1}\left(\Omega_{i}\right)$ (the latter with homogeneous Dirichlet boundary conditions) as well as the trace space $H^{1 / 2}(\Gamma)$ and the trace operator $\operatorname{tr}_{\Gamma}: H^{1}\left(\Omega_{i}\right) \rightarrow H^{1 / 2}(\Gamma), i=1, \ldots, n$, for a Lipschitz manifold $\Gamma \subset \partial \Omega_{i}$, consult, e.g., [17. Additionally, we introduce the spaces

$$
V_{i}:=\left\{v_{i} \in H^{1}\left(\Omega_{i}\right): \operatorname{tr}_{\partial \Omega \cap \partial \Omega_{i}} v_{i}=0\right\}
$$

for $i=1, \ldots, n$ and

$$
\Lambda_{i j}:=\left\{\eta \in H^{1 / 2}\left(\Gamma_{i j}\right): \eta=\operatorname{tr}_{\Gamma_{i j}} v_{i} \text { for a } v_{i} \in V_{i}\right\}=H_{00}^{1 / 2}\left(\Gamma_{i j}\right)
$$

for $|i-j|=1, i, j=1, \ldots, n$. For the same set of indices we also choose linear continuous extension operators

$$
R_{i j}: \Lambda_{i j} \rightarrow V_{i} \quad \text { and } \quad R_{j i}: \Lambda_{i j} \rightarrow V_{j}
$$

that are right-inverses to the trace operators

$$
\operatorname{tr}_{\Gamma_{i j}}: V_{i} \rightarrow \Lambda_{i j} \quad \text { and } \quad \operatorname{tr}_{\Gamma_{j i}}: V_{j} \rightarrow \Lambda_{i j}
$$

from both sides of $\Gamma_{i j}$. Despite of the non-uniqueness $\Lambda_{i j}=\Lambda_{j i}$ for $i=1, \ldots, n-1$, $j=i+1$, which we also have for the interfaces and operators above, we use this set of indices because it will simplify the notation for the algorithms in Section 4 By $(\cdot, \cdot)_{\Omega_{i}}$ we abbreviate the $L^{2}$-scalar product for scalar- and vector-valued functions on $\Omega_{i}$. We define the bi-form

$$
b_{i}\left(w_{i}, v_{i}\right):=\left(\tau_{k} K_{h, i} k r_{i}\left(w_{i}\right) \nabla w_{i}, \nabla v_{i}\right)_{\Omega_{i}} \quad \forall w_{i}, v_{i} \in H^{1}\left(\Omega_{i}\right)
$$

as well as the bilinear form

$$
a_{i}\left(w_{i}, v_{i}\right):=\left(\tau_{k} K_{h, i} \nabla w_{i}, \nabla v_{i}\right)_{\Omega_{i}} \quad \forall w_{i}, v_{i} \in H^{1}\left(\Omega_{i}\right)
$$

and the linear form

(23) $\ell_{i}\left(v_{i}\right):=\left(\bar{f}_{i}+n_{i} \theta_{i}\left(\bar{p}_{i}\right), v_{i}\right)_{\Omega_{i}}+\left(\tau_{k} K_{h, i} k r_{i}\left(\theta_{i}\left(\bar{p}_{i}\right)\right) e_{z}, \nabla v_{i}\right)_{\Omega_{i}} \quad \forall v_{i} \in H^{1}\left(\Omega_{i}\right)$.

Then in a weak formulation the multidomain problem (14) -16 in physical variables reads as follows: Find $p_{i} \in V_{i}, i=1, \ldots, n$, satisfying the subdomain equations

$$
\left(n_{i} \theta_{i}\left(p_{i}\right), v_{i}\right)_{\Omega_{i}}+b_{i}\left(p_{i}, v_{i}\right)=\ell_{i}\left(v_{i}\right) \quad \forall v_{i} \in H_{0}^{1}\left(\Omega_{i}\right), \quad i=1, \ldots n,
$$

and the weak coupling conditions

$$
\operatorname{tr}_{\Gamma_{i j}} p_{i}=\operatorname{tr}_{\Gamma_{j i}} p_{j} \quad \text { in } \Lambda_{i j}
$$




$$
\begin{aligned}
& \left(n_{i} \theta_{i}\left(p_{i}\right), R_{i j} \mu\right)_{\Omega_{i}}+b_{i}\left(p_{i}, R_{i j} \mu\right)-\ell_{i}\left(R_{i j} \mu\right)= \\
& -\left(n_{j} \theta_{j}\left(p_{j}\right), R_{j i} \mu\right)_{\Omega_{j}}-b_{j}\left(p_{j}, R_{j i} \mu\right)+\ell_{j}\left(R_{j i} \mu\right) \quad \forall \mu \in \Lambda_{i j},
\end{aligned}
$$

where (25) and (26) hold for all $i=1, \ldots, n-1$ with $j=i+1$. By defining $p:=p_{i}$ on $\Omega_{i}, i=1, \ldots, n$, and using (25) one obtains $p \in H_{0}^{1}(\Omega)$. Note that the application of Green's formula in the weak sense (see, e.g., 17]) allows to replace the functional $\ell_{i}\left(v_{i}\right)$ by the integral $\left(f_{i}, v_{i}\right)_{\Omega_{i}}$ with $f_{i}$ from (17) in (24), but not analogously in 26 .

A weak form of (18)-(20) is now obtained by Kichhoff transformation of 24 (26), in which we rewrite (23) as

$$
\ell_{i}\left(v_{i}\right)=\left(\bar{f}_{i}+n_{i} M_{i}\left(\bar{u}_{i}\right), v_{i}\right)_{\Omega_{i}}+\left(\tau_{k} K_{h, i} k r_{i}\left(M_{i}\left(\bar{u}_{i}\right)\right) e_{z}, \nabla v_{i}\right)_{\Omega_{i}} \forall v_{i} \in H^{1}\left(\Omega_{i}\right)
$$

and assume that $p_{i} \in V_{i} \Leftrightarrow u_{i} \in V_{i}$ holds with $u_{i}=\kappa_{i}\left(p_{i}\right)$. Thus our time-discrete, generalized multidomain formulation reads: Find $u_{i} \in V_{i}, i=1, \ldots, n$, satisfying the subdomain equations

$$
\left(n_{i} M_{i}\left(u_{i}\right), v_{i}\right)_{\Omega_{i}}+a_{i}\left(u_{i}, v_{i}\right)=\ell_{i}\left(v_{i}\right) \quad \forall v_{i} \in H_{0}^{1}\left(\Omega_{i}\right), \quad i=1, \ldots n,
$$

and the weak coupling conditions

$$
\begin{aligned}
& \kappa_{i}^{-1} \operatorname{tr}_{\Gamma_{i j}} u_{i}=\kappa_{j}^{-1} \operatorname{tr}_{\Gamma_{j i}} u_{j} \quad \text { in } \Lambda_{i j} \\
& \left(n_{i} M_{i}\left(u_{i}\right), R_{i j} \mu\right)_{\Omega_{i}}+a_{i}\left(u_{i}, R_{i j} \mu\right)-\ell_{i}\left(R_{i j} \mu\right)= \\
& -\left(n_{j} M_{j}\left(u_{j}\right), R_{j i} \mu\right)_{\Omega_{j}}-a_{j}\left(u_{j}, R_{j i} \mu\right)+\ell_{j}\left(R_{j i} \mu\right) \quad \forall \mu \in \Lambda_{i j},
\end{aligned}
$$

where (29) and (30) hold for all $i=1, \ldots, n-1$ with $j=i+1$. Note that in general, the subdomain functions $u_{i}$ on $\Omega_{i}, i=1, \ldots, n$, do not give rise to a global function $u \in H_{0}^{1}(\Omega)$, since in case of $\kappa_{i}^{-1} \neq \kappa_{j}^{-1}$ the coupling condition 291 induces a discontinuity of the transformed variables across the interfaces.

Equivalence $p_{i} \in V_{i} \Leftrightarrow u_{i} \in V_{i}$ holds if and only if $\kappa_{i}$ and $\kappa_{i}^{-1}$ are (for $d=1$ locally) Lipschitz continuous, see [29. This condition guarantees familiar solution spaces for $u_{i}, i=1, \ldots, n$, and trace spaces in $(29)$, but it is not satisfied for the Brooks-Corey parametrization. In this case, the inverse Kirchhoff transformations $\kappa_{i}^{-1}, i=1, \ldots, n$, even have a singularity, see Figure 4 Nevertheless, they can be used successfully in numerical simulations, see Section 5 .

Proposition 3.1. If $\theta_{i}: \mathbb{R} \rightarrow \mathbb{R}$ and $k r_{i}: \theta_{i}(\mathbb{R}) \rightarrow \mathbb{R}$ are increasing and bounded and $k r_{i}(\cdot) \geq c$ for a $c>0, i=1, \ldots, n$, then the multidomain problem (24) $-(26)$ in physical variables is equivalent to the multidomain problem (28)-(30) in generalized variables.

If the assumptions in the proposition are only satisfied for $c=0$, then one needs to require $u_{i} \in \kappa_{i}\left(V_{i}\right)$ in (24) $-(26)$ in order to maintain equivalence. For details and generalizations concerning this equivalence result we refer to [9] or [8, Sec.1.5.4]. Note, e.g., that 25$) \Leftrightarrow 29$ requires $\kappa_{i}^{-1}$ and $\operatorname{tr}_{\Gamma_{i j}}$ to commute. A substructuring result which states the equivalence of a weak global problem and its corresponding domain decomposition problem such as (14)-16) in weak form is derived in [10, for the more general case involving Signorini-type boundary conditions. That result provides a mathematical justification of the transmission conditions (25) and 26). For a generalization of these transmission conditions to domain decompositions with cross points see [14]. 
3.1.3. Well-posedness of local subproblems. Now, we shortly outline the solution theory for the subproblems 28 equipped with homogeneous Dirichlet boundary conditions on all of $\partial \Omega_{i}$. Here, the Kirchhoff transformation and our special time discretization allow us to use convex analysis.

We require $n_{i}, K_{h, i} \in L^{\infty}\left(\Omega_{i}\right), n_{i} \geq 0$ and $K_{h, i} \geq c>0$. Furthermore, let $M_{i}: \mathbb{R} \rightarrow \mathbb{R}$ and $k r_{i}:[0,1] \rightarrow \mathbb{R}$ be increasing and bounded and $\bar{f}_{i} \in L^{2}\left(\Omega_{i}\right)$ in (27). Then, with a convex primitive $\Phi_{i}$ of $M_{i}$, the functional

$$
\phi_{i}: v_{i} \mapsto \int_{\Omega_{i}} n_{i} \Phi_{i}\left(v_{i}\right) d x \quad \forall v_{i} \in H_{0}^{1}\left(\Omega_{i}\right)
$$

is proper, convex and lower semicontinuous. Furthermore, the quadratic functional

$$
\mathcal{J}_{i}: v_{i} \mapsto \frac{1}{2} a_{i}\left(v_{i}, v_{i}\right)-\ell_{i}\left(v_{i}\right) \quad \forall v_{i} \in H_{0}^{1}\left(\Omega_{i}\right)
$$

is coercive and continuous. Consequently, there exists a unique minimizer of $\phi_{i}+\mathcal{J}_{i}$ in $H_{0}^{1}\left(\Omega_{i}\right)$, see, e.g., 22. Now, finding $u_{i} \in H_{0}^{1}\left(\Omega_{i}\right)$ satisfying (28) is equivalent to determining this minimizer because $(28)$ is just the Euler-Lagrange equation of $\phi_{i}+\mathcal{J}_{i}$.

We point out that this line of thought can be generalized to cover quite general boundary conditions including Robin and Signorini-type conditions. The former lead to another convex functional on $H^{1}\left(\Omega_{i}\right)$ as long as $\kappa_{i}^{-1}: \mathbb{R} \rightarrow \mathbb{R}$ is Lipschitz continuous [10]. The latter provide a constraint to the set of solutions, so that the functional is to be minimized on a convex subset of $H_{0}^{1}\left(\Omega_{i}\right)$. In this case the variational equality (28) becomes an elliptic variational inequality of the first kind. Analogously, Brooks-Corey functions, which lead to an ill-posed $\kappa_{i}^{-1}$ with a singularity, can be considered, too, since they just add another constraint to the convex set. For further details on the solution of the time-discretized Kirchhoff-transformed Richards equation in homogeneous soil with general boundary conditions consult [13] and [8, Sec. 2.3, Thm. 3.4.2].

3.2. Fully discrete generalized multidomain formulation. In this section we describe a spatial discretization of the generalized multidomain formulation 28 (30) based on Lagrange finite elements of first order. The resulting discrete problems suggest nonlinear extensions of well-known substructuring methods because fast and robust solvers for the corresponding local convex subproblems are available 13. Special attention needs to be turned to the discretization of the second scalar product occurring in (27), since this expression contains an explicitly timediscretized convective term that has to be treated by an upwinding technique.

3.2.1. Discrete generalized multidomain problem. We assume that $\Omega$ is polygonal and that we have a triangulation $\mathcal{T}_{l}$ on $\Omega$ with the set of interior vertices $\mathcal{N}_{l}$. Here, the subscript $l$ is the discretization index. We choose Lagrange finite elements of first order on the grid, and hence $\mathcal{N}_{l}$ is also the set of all Lagrange nodes $q$ which determine the Lagrange basis $\left\{\lambda_{q}\right\}_{q \in \mathcal{N}_{l}}$ of the corresponding finite element space $\mathcal{S}_{l} \subset H_{0}^{1}(\Omega)$. Further we assume that all interfaces $\Gamma_{i j}, i=1, \ldots, n-1, j=i+1$, are resolved by the grid, and we denote by $\mathcal{N}_{l, i j}$ the set of all interior vertices located on the interface $\Gamma_{i j}$ for $i, j=1, \ldots, n, j=i \pm 1$. The finite element space $\mathcal{S}_{l, i j} \subset \Lambda_{i j}$ induced by these nodes is defined accordingly. (As in Section 3.1.2 we allow for non-uniqueness of the notation which will be helpful in Section 44) We call $\mathcal{N}_{l, i}$ the subset of all interior vertices of $\Omega_{i}$ and $\mathcal{S}_{l, i}^{0} \subset H_{0}^{1}\left(\Omega_{i}\right)$ the finite element 
space spanned by the Lagrange basis corresponding to these nodes. Furthermore, $\mathcal{S}_{l, i} \subset V_{i}$ is the finite element space spanned by the basis functions corresponding to all nodes in $\mathcal{N}_{l} \cap \bar{\Omega}_{i}$, cf. 21. We introduce the weights

$$
h_{q, i}:=\int_{\Omega_{i}} n_{i} \lambda_{q} d x \quad \forall q \in \mathcal{N}_{l}
$$

and define the extension operators

$$
R_{l, i j}: \mathcal{S}_{l, i j} \rightarrow \mathcal{S}_{l, i} \quad \text { and } \quad R_{l, j i}: \mathcal{S}_{l, i j} \rightarrow \mathcal{S}_{l, j}
$$

by zero extension to all nodes in $\mathcal{N}_{l, i}$ and $\mathcal{N}_{l, j}$, respectively. By $\ell_{l, i}\left(v_{i}\right)$ we denote an upwind finite element discretization of $\ell_{i}\left(v_{i}\right)$ to be specified in Subsection 3.2.2.

Altogether, we are now in a position to give our spatial discretization of $(18)-(20)$. It reads: Find $u_{l, i} \in \mathcal{S}_{l, i}, i=1, \ldots, n$, satisfying

$$
\sum_{q \in \mathcal{N}_{l, i}} M_{i}\left(u_{l, i}(q)\right) v_{l, i}(q) h_{q, i}+a_{i}\left(u_{l, i}, v_{l, i}\right)=\ell_{l, i}\left(v_{l, i}\right) \quad \forall v_{l, i} \in \mathcal{S}_{l, i}
$$

with discrete coupling conditions

$$
\begin{gathered}
\kappa_{i}^{-1} u_{l, i}(q)=\kappa_{j}^{-1} u_{l, j}(q) \quad \forall q \in \mathcal{N}_{l, i j} \\
\sum_{q \in \mathcal{N}_{l, i j}} M_{i}\left(u_{l, i}(q)\right) \mu_{l, i j}(q) h_{q, i}+a_{i}\left(u_{l, i}, R_{l, i j} \mu_{l, i j}\right)-\ell_{l, i}\left(R_{l, i j} \mu_{l, i j}\right)= \\
-\sum_{q \in \mathcal{N}_{l, i j}} M_{j}\left(u_{l, j}(q)\right) \mu_{l, i j}(q) h_{q, j}-a_{j}\left(u_{l, j}, R_{l, j i} \mu_{l, i j}\right)+\ell_{l, j}\left(R_{l, j i} \mu_{l, i j}\right) \\
\forall \mu_{l, i j} \in \mathcal{S}_{l, i j}
\end{gathered}
$$

where (33) and (34) hold for all $i=1, \ldots, n-1$ with $j=i+1$.

In general, as in the continuous and in the time-discrete case, the discrete functions $u_{l, i}, i=1, \ldots, n$, do not give rise to a function $u \in \mathcal{S}_{l}$ but rather to a function that is two-valued on each interface $\Gamma_{i j}, i=1, \ldots, n-1, j=i+1$. However, the coupling condition (33) enforces the continuity of the physical variables across the interfaces and can be rewritten accordingly. One can also rewrite (32) and (34) in physical variables so that the coupled problem $(32-34$ is equivalent to a finite element discretization of the time-discrete physical problem with a special quadrature for the integrals involving nonlinear terms. For details we refer to [13].

3.2.2. Upwind discretization of gravity. Finally, we turn to the discretization of $\ell_{i}\left(v_{i}\right)$ in 28$)$ and the corresponding terms in (30). By 27) the functional $\ell_{i}$ is given as a sum of two scalar products, the first of which is discretized by a quadrature rule with a sufficiently high order. The second scalar product in $\ell_{i}\left(v_{i}\right)$ reads

$$
\left(\tau_{k} K_{h, i} k r_{i}\left(M_{i}\left(\bar{u}_{i}\right)\right), \frac{\partial}{\partial z} v_{i}\right)_{\Omega_{i}} .
$$

If we only consider the time derivative and the spatial derivative $k r_{i}\left(M_{i}\left(\tilde{u}_{i}\right)\right)_{z}$ on the left hand side of the Richards equation $(9)$, we obtain a nonlinear conservation law, so that for stability reasons it is necessary to apply an upwind discretization to $k r_{i}\left(M_{i}\left(\tilde{u}_{i}\right)\right)_{z}$, consult [26]. Note that (35) is obtained from $k r_{i}\left(M_{i}\left(\tilde{u}_{i}\right)\right)_{z}$ by Green's formula and explicit time discretization. If we discretize 35 with finite elements $v_{i} \in \mathcal{S}_{l, i}$, interpolate $k r_{i}\left(M_{i}\left(\bar{u}_{i}\right)\right.$ in $\mathcal{S}_{l, i}$ and assume $K_{h, i}=$ const., this gives central differences of $-k r_{i}\left(M_{i}\left(\bar{u}_{i}\right)\right.$ in $z$-direction up to a constant in case of a regular mesh with vertical grid lines parallel to the direction of gravity. It is well 
known that by adding or substracting an artificial viscosity term, i.e., diffusion or, equivalently, central differences for the second derivative, one can obtain one-sided differences. Therefore, with a certain small weighting factor related to the mesh size, we substract a diffusion matrix in $z$-direction to the convection matrix obtained by finite element discretization of 35 in order to achieve upwinding of $-k r_{i}\left(M_{i}\left(\bar{u}_{i}\right)\right.$ in $z$-direction within the finite element framework. The CFL condition guarantees a monotone scheme, i.e., numerical stability, in the linear case if time step sizes are chosen sufficiently small. Further details can be found in [8, Sec. 4.2.1] and in [13.

3.2.3. Minimization formulation for discrete local subproblems. The sums in (32) and (34) represent a discretization of the corresponding integrals in (28) and (30) by Lagrange interpolation of the product $M_{i}\left(u_{i}\right) v_{i}$, respectively. They can be regarded as a lumping of the $L^{2}$-scalar product and represent the Fréchet derivative of a discretization of the convex functional $\phi_{i}$ in (31) in which $\Phi_{i}\left(v_{i}\right)$ is interpolated by Langrange functions. Therefore, with homogeneous Dirichlet boundary conditions for instance, as in the continuous case, each finite dimensional subproblem 32 for $i=1, \ldots, n$ is equivalent to a uniquely solvable minimization problem for a convex functional on $\mathcal{S}_{l, i}$. This result can be extended to the use of Brooks-Corey parameter functions and quite general Dirichlet, Neumann, Robin and Signorinitype boundary conditions. Furthermore, for homogeneous problems, convergence of the discrete solutions to the continuous solution for decreasing mesh size can be shown in the Brooks-Corey case and for general boundary conditions [8, 13, 15.

3.3. Multidomain discretization in physical variables via local inverse discrete Kirchhoff transformation. Observe that one can rewrite the discrete interface condition (33) more similar to its continuous counterpart (29) if one considers the Lagrange interpolation operator

$$
I_{\mathcal{S}_{l, i j}}: \Lambda_{i j} \cap C\left(\Gamma_{i j}\right) \rightarrow \mathcal{S}_{l, i j}
$$

defined by $\left(I_{\mathcal{S}_{l, i j}} \mu\right)(q)=\mu(q)$ for all $q \in \mathcal{N}_{l, i j}$ and all relevant indices $i, j, l$. Then (33) reads

$$
I_{\mathcal{S}_{l, i j}} \kappa_{i}^{-1}\left(\left.u_{l, i}\right|_{\Gamma_{i j}}\right)=I_{\mathcal{S}_{l, i j}} \kappa_{j}^{-1}\left(\left.u_{l, j}\right|_{\Gamma_{i j}}\right) .
$$

Analogously we define the interpolation operator

$$
I_{\mathcal{S}_{l, i}}: V_{i} \cap C\left(\bar{\Omega}_{i}\right) \rightarrow \mathcal{S}_{l, i}
$$

given by $\left(I_{\mathcal{S}_{l, i}} v\right)(q)=v(q)$ for all $q \in \mathcal{N}_{l, i}$ and all relevant indices $i, l$.

Definition 3.2. We call $I_{\mathcal{S}_{l, i}} \kappa_{i}: \mathcal{S}_{l, i} \rightarrow \mathcal{S}_{l, i}$ discrete Kirchhoff transformation on $\mathcal{S}_{l, i}$, and $p_{l} \in \mathcal{S}_{l}$ given by

$$
p_{l, i}=I_{\mathcal{S}_{l, i}} \kappa_{i}^{-1} u_{l, i}, \quad i=1, \ldots, n,
$$

discrete physical pressure associated with the discrete generalized multidomain problem (32) - 34.

The inverse discrete Kirchhoff transformation on $\mathcal{S}_{l, i}$ is $I_{\mathcal{S}_{l, i}} \kappa_{i}^{-1}$, and $I_{\mathcal{S}_{l, i j}} \kappa_{i}^{-1}$ as well as $I_{\mathcal{S}_{l, i j}} \kappa_{j}^{-1}$ in 36 can be regarded as inverse discrete Kirchhoff transformations on $\mathcal{S}_{l, i j}$ corresponding to $\Omega_{i}$ and $\Omega_{j}$, respectively.

Now, just as the continuous multidomain probem $(28)-(30)$ in generalized variables arose from Kirchhoff transformation of the corresponding problem (24)-(26) in physical pressure, one can obtain a discrete multidomain problem in physical 
pressure by inverse discrete Kirchhoff transformation of the corresponding discrete problem 32 - 34 in generalized variables. This finally amounts to a new discretization of the physical multidomain problem (24)-26.

In fact, by definition of $M_{i}, i=1, \ldots, n$, in $(8)$, the terms in the sums as well as in the linear functionals $\ell_{l, i}, i=1, \ldots, n$, cf. (28) and (30), can be easily rewritten in discrete physical pressure. Discrete Kirchhoff transformation of 29 follows directly from (37). The reformulation of the bilinear forms $a_{i}(\cdot, \cdot)$ in terms of $p_{l, i}$, however, is not straightforward. In fact, just as $a_{i}(\cdot, \cdot)$ arose from $b_{i}(\cdot, \cdot)$ by the chain rule (7) applied to the Kirchhoff transformation in the continuous setting, a kind of discrete chain rule involving the discrete Kirchhoff transformation is needed now. Such a discrete chain rule was derived in 13 using the mean value theorem. It reads as follows.

Lemma 3.3 (Discrete chain rule). Let $\theta_{i}: \mathbb{R} \rightarrow \mathbb{R}$ and $k r_{i}: \theta_{i}(\mathbb{R}) \rightarrow \mathbb{R}$ be increasing and continuous and $k r_{i}(\cdot)>0$ for $i=1, \ldots, n$. Let $t \in \mathcal{T}_{l}$ be a simplex of the grid on $\Omega_{i}$ given by edges $e_{k}, k=1, \ldots, d$, that meet in a point. Furthermore, let $p_{l, i}$ and $u_{l, i}=I_{\mathcal{S}_{l, i}} \kappa_{i}\left(p_{l, i}\right)$ in $\mathcal{S}_{l, i}$ be given for $i=1, \ldots, n$. Then there exist points $x_{k}$, $k=1, \ldots, d$, each located on the edge $e_{k}$, and depending on $\kappa_{i}$ and $p_{l, i}$ such that the discrete chain rule

$$
\nabla u_{l, i}=D_{t}\left(p_{l, i}\right) \nabla p_{l, i} \quad \text { on } t
$$

holds with the diagonal matrix

$$
D_{t}\left(p_{l, i}\right)=\operatorname{diag}\left(k r_{i}\left(\theta_{i}\left(p_{l, i}\left(x_{1}\right)\right)\right), \ldots, k r_{i}\left(\theta_{i}\left(p_{l, i}\left(x_{d}\right)\right)\right)\right) .
$$

Now we can introduce the bi-form

$$
b_{l, i}\left(w_{l, i}, v_{l, i}\right):=\sum_{t \in \mathcal{T}_{l, i}} \int_{t} D_{t}\left(w_{l, i}\right) \nabla w_{l, i} \nabla v_{l, i} d x \quad \forall w_{l, i}, v_{l, i} \in \mathcal{S}_{l, i}
$$

for $i=1, \ldots, n$, which can be regarded as an inexact version of $b_{i}(\cdot, \cdot)$ given in $(22)$, with a quadrature rule involving the quadrature points given by Lemma 3.3 .

Altogether, local Kirchhoff transformation of the weak, time-discrete multidomain formulation (24)-26), discretization of the resulting generalized formulation by finite elements, and local inverse Kirchhoff transformation (37) provides the desired multidomain discretization of the Richards equation. It can be regarded as a finite element discretization of the physical multidomain formulation (24)-(26) with nonstandard quadrature and it reads: Find $p_{l, i} \in \mathcal{S}_{l, i}, i=1, \ldots, n$, satisfying

$$
\sum_{q \in \mathcal{N}_{l, i}} \theta_{i}\left(p_{l, i}(q)\right) v_{l, i}(q) h_{q, i}+b_{l, i}\left(p_{l, i}, v_{l, i}\right)=\ell_{l, i}\left(v_{l, i}\right) \quad \forall v_{l, i} \in \mathcal{S}_{l, i}
$$

with discrete coupling conditions

$$
\begin{gathered}
\sum_{q \in \mathcal{N}_{l, i j}} \theta_{i}\left(p_{l, i}(q)\right) \mu_{l, i j}(q) h_{q, i}+b_{l, i}\left(p_{l, i}, R_{l, i j} \mu_{l, i j}\right)-\ell_{l, i}\left(R_{l, i j} \mu_{l, i j}\right)= \\
-\sum_{q \in \mathcal{N}_{l, i j}} \theta_{j}\left(p_{l, j}(q)\right) \mu_{l, i j}(q) h_{q, j}-b_{l, j}\left(p_{l, j}, R_{l, j i} \mu_{l, i j}\right)+\ell_{l, j}\left(R_{l, j i} \mu_{l, i j}\right) \\
\forall \mu_{l, i j} \in \mathcal{S}_{l, i j}
\end{gathered}
$$


where (39) and 40) hold for all $i=1, \ldots, n-1$ with $j=i+1$. Analogously as in the continuous case (24)-26], we obtain $p_{l} \in \mathcal{S}_{l}$ by defining $p_{l}:=p_{l, i}$ on $\Omega_{i}$, $i=1, \ldots, n$, and using (39).

We close this section with the discrete counterpart of Proposition 3.1

Proposition 3.4. If $\theta_{i}: \mathbb{R} \rightarrow \mathbb{R}$ and $k r_{i}: \theta_{i}(\mathbb{R}) \rightarrow \mathbb{R}$ are increasing and continuous and $k r_{i}(\cdot)>0, i=1, \ldots, n$, then the discrete multidomain problem (24)-(26) in physical variables is equivalent to the multidomain problem (38)-40) in generalized variables. The solutions of the two discrete formulations are related by inverse discrete Kirchhoff transformation (37).

While (suboptimal) convergence results are available for homogeneous parameter functions [13, related results in the present heterogeneous case are still open.

\section{Algebraic solution by nOnlinear substructuring methods}

4.1. Nonlinear Dirichlet-Neumann method. Our version of the DirichletNeumann method is motivated by the chequerboard ansatz in [31, Sec. 1.4.2]. We first apply it to the continuous multidomain problem (28)-(30) and then to the discrete one (32)-(34). In addition, we state some theoretical results.

4.1.1. The continuous case. As in the previous section, we give a formulation of the method for the multidomain problem (28)-(30) with homogeneous boundary conditions on $\partial \Omega$. Therefore, we seek subdomain solutions in the spaces $V_{i}$ given in 21. We choose a global damping parameter $\vartheta \in(0,1)$ although one could also choose different ones for each interface. As the initial iterates $u_{i}^{0} \in V_{i}, i=1, \ldots, n$, we take the subdomain solutions from the previous time step. Then, with known iterates $u_{i}^{k} \in V_{i}$ for $k \geq 0$ we obtain the new iterates $u_{i}^{k+1} \in V_{i}$ by first solving

$$
\begin{gathered}
\left(n_{i} M_{i}\left(u_{i}^{k+1}\right), v_{i}\right)_{\Omega_{i}}+a_{i}\left(u_{i}^{k+1}, v_{i}\right)=\ell_{i}\left(v_{i}\right) \quad \forall v_{i} \in H_{0}^{1}\left(\Omega_{i}\right), \quad \forall i \text { odd }, \\
\operatorname{tr}_{\Gamma_{i j}} u_{i}^{k+1}=\kappa_{i} \kappa_{j}^{-1}\left(\vartheta \operatorname{tr}_{\Gamma_{j i}} u_{j}^{k}+(1-\vartheta) \kappa_{j} \kappa_{i}^{-1} \operatorname{tr}_{\Gamma_{i j}} u_{i}^{k}\right) \quad \text { in } \Lambda_{i j}, \\
j=i \pm 1 \in\{1, \ldots, n\},
\end{gathered}
$$

and then

$$
\begin{aligned}
& \left(n_{i} M_{i}\left(u_{i}^{k+1}\right), v_{i}\right)_{\Omega_{i}}+a_{i}\left(u_{i}^{k+1}, v_{i}\right)=\ell_{i}\left(v_{i}\right) \quad \forall v_{i} \in H_{0}^{1}\left(\Omega_{i}\right), \quad \forall i \text { even, } \\
& \left(n_{i} M_{i}\left(u_{i}^{k+1}\right), R_{i j} \mu\right)_{\Omega_{i}}+a_{i}\left(u_{i}^{k+1}, R_{i j} \mu\right)-\ell_{i}\left(R_{i j} \mu\right)= \\
& \quad-\left(n_{j} M_{j}\left(u_{j}^{k+1}\right), R_{j i} \mu\right)_{\Omega_{j}}-a_{j}\left(u_{j}^{k+1}, R_{j i} \mu\right)+\ell_{j}\left(R_{j i} \mu\right) \quad \forall \mu \in \Lambda_{i j}, \\
& \quad j=i \pm 1 \in\{1, \ldots, n\} .
\end{aligned}
$$

Note that we carry out the damping of Dirichlet values on $\Gamma_{i j}$ in terms of the transformed variables corresponding to $\Omega_{j}$. Also, observe that in each iteration step inverse Kirchhoff transformations $\kappa_{i}^{-1}, \kappa_{j}^{-1}$ have to be evaluated on the interfaces $\Gamma_{i j}$. Hence, in contrast to the homogeneous case, the typical ill-conditioning of the Richards equation (cf. Figure 4 is no longer separated from the algebraic solution process. 
4.1.2. Convergence properties. In 1D the nonlinear Dirichlet-Neumann method for $n=2$ converges for the stationary Richards equation without gravity in case of sufficiently small damping parameter if we have $c \leq K_{h, i} k r(\cdot) \leq C$ for some positive constants $c, C$ and Lipschitz continuous $K_{h, i}: \Omega_{i} \rightarrow \mathbb{R}$, see [8, 11]. In higher dimensions convergence can be ensured if the Kirchhoff transformations $\kappa_{i}, i=1,2$, and their inverses are Lipschitz continuous superposition operators in the trace space and certain smallness conditions on their norms are satisfied.

4.1.3. The discrete case. Now we turn to the discrete version of (41)-44), i.e., to our version of the Dirichlet-Neumann method for the fully discrete multidomain problem $(32-(34)$. Again we choose a global damping parameter $\vartheta \in(0,1)$ and the subdomain solutions from the previous time step as the initial iterates $u_{l, i}^{0} \in \mathcal{S}_{l, i}$, $i=1, \ldots, n$. Then, with known iterates $u_{l, i}^{k} \in \mathcal{S}_{l, i}$ for $k \geq 0$ we determine the new iterates $u_{i}^{k+1} \in \mathcal{S}_{l, i}$ by first solving for odd $i$ :

$$
\begin{gathered}
\sum_{q \in \mathcal{N}_{l, i}} M_{i}\left(u_{l, i}^{k+1}(q)\right) v_{l, i}(q) h_{q, i}+a_{i}\left(u_{l, i}^{k+1}, v_{l, i}\right)=\ell_{l, i}\left(v_{l, i}\right) \quad \forall v_{l, i} \in \mathcal{S}_{l, i}^{0} \\
u_{l, i}^{k+1}(q)=\kappa_{i} \kappa_{j}^{-1}\left(\vartheta u_{l, j}^{k}(q)+(1-\vartheta) \kappa_{j} \kappa_{i}^{-1} u_{l, i}^{k}(q)\right) \quad \\
\quad \forall q \in \mathcal{N}_{l, i j}, \\
j=i \pm 1 \in\{1, \ldots, n\},
\end{gathered}
$$

and then for even $i$ :

$$
\begin{gathered}
\sum_{q \in \mathcal{N}_{l, i}} M_{i}\left(u_{l, i}^{k+1}(q)\right) v_{l, i}(q) h_{q, i}+a_{i}\left(u_{l, i}^{k+1}, v_{l, i}\right)=\ell_{l, i}\left(v_{l, i}\right) \quad \forall v_{l, i} \in \mathcal{S}_{l, i}^{0} \\
\sum_{q \in \mathcal{N}_{l, i j}} M_{i}\left(u_{l, i}^{k+1}(q)\right) \mu_{l, i j}(q) h_{q, i}+a_{i}\left(u_{l, i}^{k+1}, R_{l, i j} \mu_{l, i j}\right)-\ell_{l, i}\left(R_{l, i j} \mu_{l, i j}\right)= \\
-\sum_{q \in \mathcal{N}_{l, i j}} M_{j}\left(u_{l, j}^{k+1}(q)\right) \mu_{l, i j}(q) h_{q, j}-a_{j}\left(u_{l, j}^{k+1}, R_{l, j i} \mu_{l, i j}\right)+\ell_{l, j}\left(R_{l, j i} \mu_{l, i j}\right) \\
\forall \mu_{l, i j} \in \mathcal{S}_{l, i j}, \quad j=i \pm 1 \in\{1, \ldots, n\} .
\end{gathered}
$$

What has been pointed out in Subsection 4.1.2 for the continuous version of the algorithm applies analogously to the discrete version. In particular, the convergence results carry over to the discrete case with the same constants which entails mesh-independent convergence rates in $1 D$ with $n=2$. Indeed, asymptotic meshindependence of convergence rates and corresponding optimal damping parameters can also be observed in numerical examples in 2D. In the case with two subdomains, the convergence rates improve if extremely different soil types (with $K_{h, 1}$ and $K_{h, 2}$ differing by some orders of magnitude) are used in $\Omega_{1}$ and $\Omega_{2}$ as long as the domain with the higher permeability is chosen as the Neumann domain. See 12 for some numerical studies on this topic.

4.2. Nonlinear Robin method. Similarly as for the Dirichlet-Neumann method we now introduce and discuss our version of a nonlinear Robin algorithm applied to the non-overlapping domain decomposition problem $\sqrt{18}-(20)$ and its discrete counterpart $32-34$. 
4.2.1. The continuous case. Robin conditions are linear combinations of Dirichlet and Neumann boundary conditions. Roughly speaking, for a $\gamma>0$, the Robin method results in an iteration between two subdomains over the sum $\mathbf{v} \cdot \mathbf{n}+\gamma p=$ $\mathbf{v} \cdot \mathbf{n}+\gamma \kappa^{-1} u$ across the interface. This sum, intended to be equal from both neighbouring subdomains, is multiplied with test functions and integrated over the interface, followed by the application of Green's formula to $\mathbf{v} \cdot \mathbf{n}$ on both subdomains meeting at that interface.

On our layered decomposition of $\Omega$ this gives rise to the following sequential algorithm that treats the subdomains from the top to the bottom. We choose a relaxation parameter $\gamma_{i}>0$ for each $\Omega_{i}, i=1, \ldots, n$, and by $(\cdot, \cdot)_{\Gamma_{i j}}$ abbreviate the $L^{2}$-scalar product on $\Gamma_{i j}, j=i \pm 1$. As above, we take the subdomain solutions from the previous time step as the initial iterates $u_{i}^{0} \in V_{i}, i=1, \ldots, n$. Then with known iterates $u_{i}^{k} \in V_{i}$ for a $k \geq 0$ we generate the new iterates $u_{i}^{k+1} \in V_{i}$ by solving successively for $i=1, \ldots, n$ the Robin subdomain problems

$$
\begin{gathered}
\left(n_{i} M_{i}\left(u_{i}^{k+1}\right), v_{i}\right)_{\Omega_{i}}+a_{i}\left(u_{i}^{k+1}, v_{i}\right)=\ell_{i}\left(v_{i}\right) \quad \forall v_{i} \in H_{0}^{1}\left(\Omega_{i}\right) \\
\left(n_{i} M_{i}\left(u_{i}^{k+1}\right), R_{i j} \mu\right)_{\Omega_{i}}+a_{i}\left(u_{i}^{k+1}, R_{i j} \mu\right)-\ell_{i}\left(R_{i j} \mu\right)+\gamma_{i}\left(\kappa_{i}^{-1} u_{i}^{k+1}, \mu\right)_{\Gamma_{i j}} \\
=-\left(n_{j} M_{j}\left(u_{j}^{k+1}\right), R_{j i} \mu\right)_{\Omega_{j}}-a_{j}\left(u_{j}^{k+1}, R_{j i} \mu\right)+\ell_{j}\left(R_{j i} \mu\right)+\gamma_{i}\left(\kappa_{j}^{-1} u_{j}^{k+1}, \mu\right)_{\Gamma_{i j}} \\
\forall \mu \in \Lambda_{i j}, j=i-1 \in\{1, \ldots, n\}, \\
\left(n_{i} M_{i}\left(u_{i}^{k+1}\right), R_{i j} \mu\right)_{\Omega_{i}}+a_{i}\left(u_{i}^{k+1}, R_{i j} \mu\right)-\ell_{i}\left(R_{i j} \mu\right)+\gamma_{i}\left(\kappa_{i}^{-1} u_{i}^{k+1}, \mu\right)_{\Gamma_{i j}} \\
=-\left(n_{j} M_{j}\left(u_{j}^{k}\right), R_{j i} \mu\right)_{\Omega_{j}}-a_{j}\left(u_{j}^{k}, R_{j i} \mu\right)+\ell_{j}\left(R_{j i} \mu\right)+\gamma_{i}\left(\kappa_{j}^{-1} u_{j}^{k}, \mu\right)_{\Gamma_{i j}} \\
\forall \mu \in \Lambda_{i j}, j=i+1 \in\{1, \ldots, n\} .
\end{gathered}
$$

As the Dirichlet-Neumann method the Robin method can also be carried out for more general boundary conditions on $\partial \Omega$ like in the hydrological example in Section 5.3. Moreover, since Robin conditions lead to uniquely solvable subdomain problems (see Theorem 4.1 for the conditions and [15] for more details) we do not encounter non-uniqueness issues as they could occur in the Dirichlet-Neumann method. As for the latter in (41)-(44), we could also consider a parallelizable version of the Robin method in which we first treat all subdomains for odd indices $i$, calculating (49) with (51) also for $j-1 \in\{1, \ldots, n\}$, and then for even indices $i$, calculating (49) with (50) also for $j+1 \in\{1, \ldots, n\}$.

As for the Dirichlet-Neumann method, the interface conditions require the evaluation of ill-conditioned, inverse Kirchhoff transformations $\kappa_{i}^{-1}, \kappa_{j}^{-1}$ in each step of the Robin iteration.

4.2.2. Convergence properties. We can state the following result for $n=2$ subdomains and homogeneous Dirichlet boundary conditions [8].

Theorem 4.1. In $1 D$ and with two subdomains the Robin method 499-51) converges if $\gamma_{1}=\gamma_{2}$ and, for $i=1,2, M_{i}: \mathbb{R} \rightarrow \mathbb{R}$ are Lipschitz continuous increasing functions and $c \leq K_{h, i} k r(\cdot) \leq C$ while $n_{i}$ are positive functions in $C\left(\bar{\Omega}_{i}\right)$ and the $K_{h, i}: \Omega_{i} \rightarrow \mathbb{R}$ are Lipschitz continuous.

A more general analysis including a convergence result for certain degenerate $k r$, i.e., with the property $k r(\theta) \rightarrow 0$ for $\theta \rightarrow \theta_{m}$, is feasible. 
4.2.3. The discrete case. We discretize 49 -51) in the way explained in Subsection 3.2 in order to obtain our Robin method for the discrete multidomain problem (32)-(34). The discretization of all terms has appeared above, with the exception of the last integral $\left(\kappa_{j}^{-1} \cdot \mu\right)_{\Gamma_{i j}}$ in the Robin conditions (50) and (51). In accordance with the treatment of the first integrals in 49 over the subdomains, the integrals over the interface $\Gamma_{i j}$ are discretized via Lagrange interpolation of the integrands. This means, with the weights

$$
h_{q, i j}:=\int_{\Gamma_{i j}} \lambda_{q} d x \quad \forall q \in \mathcal{N}_{l, i j}
$$

we discretize

$$
\left(\kappa_{i}^{-1} u_{i}^{k+1}, \mu\right)_{\Gamma_{i j}} \quad \text { by } \quad \sum_{q \in \mathcal{N}_{l, i j}} \kappa_{i}^{-1}\left(u_{l, i}^{k+1}(q)\right) \mu_{l, i j}(q) h_{q, i j} .
$$

For first-order finite element basis functions $\lambda_{q}$, the $h_{q, i j}$ are positive and the discrete functions

$$
u_{i}^{k+1} \mapsto \sum_{q \in \mathcal{N}_{l, i j}} \kappa_{i}^{-1}\left(u_{l, i}^{k+1}(q)\right) \mu_{l, i j}(q) h_{q, i j}
$$

are increasing.

Altogether, again with initial iterates $u_{i}^{0} \in \mathcal{S}_{l, i}$ coming from the solution of the previous time step, we obtain the following scheme for the solution of (32)-(34). With $\gamma_{i}>0$ for $i=1, \ldots, n$, and known $u_{i}^{k} \in \mathcal{S}_{l, i}$ for a $k \geq 0$ we obtain the new iterates $u_{i}^{k+1} \in \mathcal{S}_{l, i}$ by solving the discrete Robin subdomain problems

$$
\begin{aligned}
& \sum_{q \in \mathcal{N}_{l, i j}} M_{i}\left(u_{l, i}^{k+1}(q)\right) \mu_{l, i j}(q) h_{q, i}+a_{i}\left(u_{l, i}^{k+1}, R_{l, i j} \mu_{l, i j}\right)-\ell_{l, i}\left(R_{l, i j} \mu_{l, i j}\right) \\
& \quad+\gamma_{i} \sum_{q \in \mathcal{N}_{l, i j}} \kappa_{i}^{-1}\left(u_{l, i}^{k+1}(q)\right) \mu_{l, i j}(q) h_{q, i j}= \\
& \quad-\sum_{q \in \mathcal{N}_{l, i j}} M_{j}\left(u_{l, j}^{k}(q)\right) \mu_{l, i j}(q) h_{q, j}-a_{j}\left(u_{l, j}^{k}, R_{l, j i} \mu_{l, i j}\right)+\ell_{l, j}\left(R_{l, j i} \mu_{l, i j}\right) \\
& +\gamma_{i} \sum_{q \in \mathcal{N}_{l, i j}} \kappa_{j}^{-1}\left(u_{l, j}^{k}(q)\right) \mu_{l, i j}(q) h_{q, i j} \quad \forall \mu_{l, i j} \in \mathcal{S}_{l, i j}, j=i+1 \in\{1, \ldots, n\} .
\end{aligned}
$$

As in the continuous case the discrete Robin problems are uniquely solvable, even in the case of Brooks-Corey parameter functions that do not satisfy the conditions 
in Theorem 4.1, see [8, p. 194]. For the solution theory and the application of multigrid to Robin problems of this kind we refer to [10] and [8, Sec. 3.4].

With the conditions stated in Theorem 4.1 for the continuous case we can also show convergence of the discrete Robin method in 1D [8, Thm. 3.4.30]. Numerically, we also observe convergence in higher space dimensions and even for the Brooks-Corey parameter functions, see Subsection 5.3 or [10]. In contrast to the Dirichlet-Neumann method, we cannot prove mesh-independence. This is no surprise, as the Robin method is known to be mesh-dependent even for linear problems. Instead, the actual asymptotic behaviour of the degenerating convergence rates and corresponding optimal relaxation parameters with respect to the mesh size have been determined theoretically and experimentally for many linear problems, see [25, 28. We were able to confirm many of those relationships numerically in our nonlinear cases, too. The results of those numerical studies can be found in $[12$.

\section{NuMERICAL EXPERIMENTS}

5.1. Discretization error. To numerically assess the spatial discretization error of our multidomain discretization, we construct a model problem (14 -16$)$ on $n=2$ subdomains with known closed-form solution. We omit additional source terms and the gravity term for simplicity, i.e., we set $f_{i}=n_{i} \theta_{i}\left(\bar{p}_{i}\right), f_{i j}=f_{j i}=0$, and $n_{i}=\tau_{k}=1$. In order to capture typical challenges of the given problem, we consider a solution with saturated and unsaturated parts in each subdomain, and such that the saturation front, i.e., the border between these parts, crosses the interface.

The computational domain $\Omega=(-1,1)^{2}$ is divided into two subdomains $\Omega_{1}=$ $(-1,1) \times(0,1)$ and $\Omega_{2}=(-1,1) \times(-1,0)$, which meet at the interface $\Gamma=\bar{\Omega}_{1} \cap \bar{\Omega}_{2}=$ $(-1,1) \times\{0\}$ (meters are used as the unit of length). On $\Omega$ we make the ansatz

$$
p(x, y)=p_{i}(x, y) \quad \text { in } \Omega_{i},
$$

and

$$
p_{i}(x, y)=\omega(x)+a g_{i}(y) K_{h, i}^{-1} k r_{i}\left(\theta_{i}(\omega(x))\right)^{-1}, \quad i=1,2 .
$$

Here, $\omega, g_{i} \in C^{1}(\mathbb{R})$ are functions to be specified below, $K_{h, 1}, K_{h, 2}$ are the hydraulic conductivities in the subdomains, $\theta_{i}$ and $k r_{i}$ are the parameter functions (2) and (3), respectively, and $a \in \mathbb{R}$ is a scaling parameter.

To obtain the desired behaviour we set

$$
\omega(x)= \begin{cases}p_{b, 1}-\left(p_{b, 2}-p_{b, 1}\right)\left(\frac{x-\xi_{1}}{\Delta \xi}\right)^{2} & \text { if } x \leq \xi_{1}, \\ p_{b, 1}+\left(p_{b, 2}-p_{b, 1}\right)\left(\frac{x-\xi_{2}}{\Delta \xi}-1\right)^{2}\left(\frac{x-\xi_{2}}{\Delta \xi}+1\right)^{2} & \text { if } \xi_{1} \leq x \leq \xi_{2}, \\ p_{b, 2}+\left(p_{b, 2}-p_{b, 1}\right)\left(\frac{x-\xi_{2}}{\Delta x}\right)^{2} & \text { if } \xi_{2} \leq x,\end{cases}
$$

where $-1<\xi_{1}<\xi_{2}<1$ are two parameters and $\Delta \xi=\xi_{2}-\xi_{1}$. We assume that $p_{b, 2}>p_{b, 1}$. For $g_{i}$ we select

$$
g_{1}(y)=\sin (2 \pi y) \quad \text { and } \quad g_{2}(y)=2 \pi y .
$$

Note that $g_{1}(0)=g_{2}(0)$, and hence $p$ is continuous across $\Gamma$. Also, $g_{1}^{\prime}(0)=g_{2}^{\prime}(0)$, and hence the flux continuity condition (16)

$$
K_{h, 1} k r_{1}\left(\theta_{1}\left(p_{1}(x, 0)\right)\right) \frac{\partial}{\partial y} p_{1}(x, 0)=K_{h, 2} k r_{2}\left(\theta_{2}\left(p_{2}(x, 0)\right)\right) \frac{\partial}{\partial y} p_{2}(x, 0)
$$




\begin{tabular}{|c||c|c|c|c|c|c|}
\hline & $n$ & $\theta_{m}$ & $\theta_{M}$ & $\lambda$ & $p_{b}[\mathrm{~m}]$ & $K_{h}[\mathrm{~m} / \mathrm{s}]$ \\
\hline \hline sand & 0.437 & 0.0458 & 1 & 0.694 & -0.0726 & $6.54 \cdot 10^{-5}$ \\
\hline loam & 0.463 & 0.027 & 1 & 0.252 & -0.1115 & $3.67 \cdot 10^{-6}$ \\
\hline
\end{tabular}

TABLE 1. Soil parameters

holds as well. We check that the saturation front crosses the interface. Recall from (2) that $(x, y) \in \Omega_{i} \cup \Gamma$ is on the saturation front if $p(x, y)=p_{b, i}$ and $p(\tilde{x}, \tilde{y})<$ $p_{b, i}$ for some $(\tilde{x}, \tilde{y})$ in every neighbourhood of $(x, y)$ in $\Omega_{i} \cup \Gamma$. By construction we have $p(x, 0)=\omega(x)$ and $\omega(x)=p_{b, i}$ if $x=\xi_{i}$. From the definition of $\omega$ we see that $\omega(x)<p_{b, 1}$ if $x<\xi_{1}, \omega(x)>p_{b, 2}$ if $x>\xi_{2}$, and $p_{b, 1}<\omega(x)<p_{b, 2}$ for $\xi_{1}<x<\xi_{2}$. Hence the saturation front intersects $\Gamma$ on at least $\left[\xi_{1}, \xi_{2}\right] \times\{0\}$.

We finally discuss the smoothness of $p$. Note that the derivative

$$
\left(k r_{i}\left(\theta_{i}(\omega(x))\right)^{\prime}=\left\{\begin{array}{cl}
-\left(2+3 \lambda_{i}\right)\left(\frac{\omega(x)}{p_{b, i}}\right)^{-3-3 \lambda_{i}} \frac{\omega^{\prime}(x)}{p_{b, i}} & \text { if } x \leq \xi_{i}, \\
0 & \text { if } x>\xi_{i},
\end{array}\right.\right.
$$

is continuous, since $\omega\left(\xi_{i}\right)=p_{b, i}$ and $\omega^{\prime}\left(\xi_{i}\right)=0$, and piecewise differentiable with respect to $x$. From this we conclude that

$$
\frac{\partial}{\partial x} p_{i}(x, y)=\omega^{\prime}(x)-g_{i}(y) K_{h, i}^{-1} k r_{i}\left(\theta _ { i } ( \omega ( x ) ) ^ { - 2 } \left(k r_{i}\left(\theta_{i}(\omega(x))\right)^{\prime}\right.\right.
$$

is also continuous and piecewise differentiable with respect to $x$ and $y$. Continuity and piecewise differentiability of $\frac{\partial}{\partial y} p_{i}(x, y)$ with respect to $x$ and $y$ is also easy to verify. Hence $p_{i} \in H^{2}\left(\Omega_{i}\right)$ for both subdomains. Since the $p_{i}$ are continuous across $\Gamma$, we get $p \in H^{1}(\Omega)$ for the entire domain. However, since the normal derivative jumps across $\Gamma$, we get $p \notin H^{2}(\Omega)$. Analogously, since $k r_{i}\left(\theta_{i}\left(p_{i}\right)\right) \nabla p_{i}$ is continuous and the classical derivatives exist piecewise, the weak derivatives of $k r_{i}\left(\theta_{i}\left(p_{i}\right)\right) \nabla p_{i}$ coincide with the strong ones wherever those exist. Hence the right hand sides $f_{i} \in L^{2}\left(\Omega_{i}\right)$ can be computed pointwise in $\Omega_{i}$ as

$$
f_{i}:=\theta_{i}\left(p_{i}\right)-\operatorname{div}\left(K_{h, i} k r_{i}\left(\theta_{i}\left(p_{i}\right) \nabla p_{i}\right), \quad i=1,2 .\right.
$$

Denote by $p_{\partial}$ the trace of $p$ on $\partial \Omega$. Then, $p$ is a solution of our benchmark problem

$$
\begin{aligned}
\theta_{i}\left(p_{i}\right)-\operatorname{div}\left(K_{h, i} k r_{i}\left(\theta_{i}\left(p_{i}\right) \nabla p_{i}\right)\right. & =f_{i} & & \text { in } \Omega_{i}, i=1,2, \\
p & =p_{\partial} & & \text { on } \partial \Omega .
\end{aligned}
$$

We picked soil parameters corresponding to sand in $\Omega_{1}$ and loam in $\Omega_{2}$ as given in 32 and listed in Table 1. We set $\xi_{1}=-0.4, \xi_{2}=0.4$, and the scaling parameter $a=10^{-7}$. With these data, Figure 6 shows the solution $p$ on the left with the range $[-0.178 \mathrm{~m},-0.023 \mathrm{~m}]$ on $\Omega_{1}$ and $[-0.248 \mathrm{~m},-0.051 \mathrm{~m}]$ on $\Omega_{2}$. The size of the unsaturated regimes and a comparison of the range of $p$ with the bubbling pressures in Table 1 make clear that we have chosen a nonlinear problem in which the linear, i.e., saturated regimes do not seem to dominate. The corresponding source term $f$ with $f_{\mid \Omega_{i}}=f_{i}$ is depicted on the right in Figure 6. Note that this is a challenging numerical problem, as the source term $f$ is not continuous on the subdomains and features extreme "ridges", which are difficult to integrate numerically. We use a 

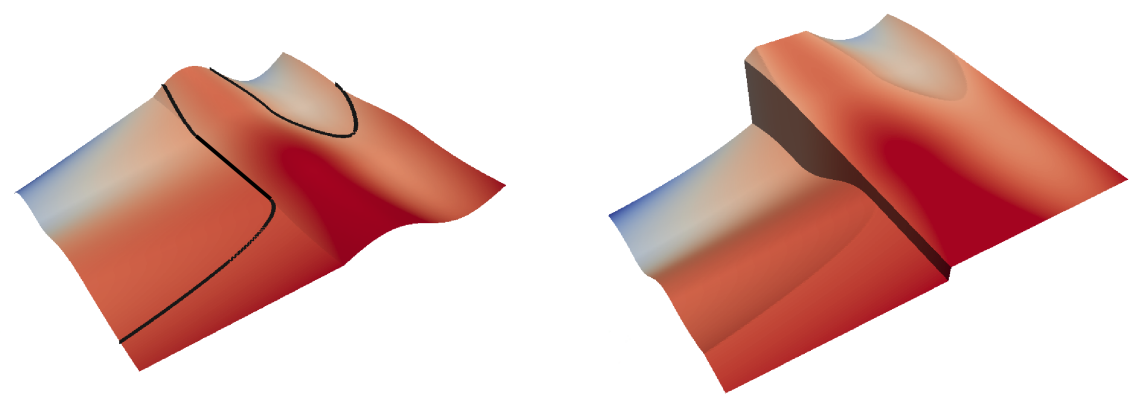

FiguRE 6. Left: solution $p$ of the benchmark problem (black lines: saturation front). Right: corresponding source term $f$. Subdomain $\Omega_{2}$ is on the left.

composite quadrature rule obtained by uniformly refining each element three times, and using a fifth-order quadrature on each subelement.

We discretized the domain $\Omega$ with a uniform triangle grid on $3 \times 3$ vertices, and constructed a sequence of test grids by up to 10 steps of uniform refinement. We use the Dune libraries ${ }^{1}[5$, for the implementation, and DUNE-GRID-GLue [6] to handle the grid coupling. For each grid we solved the problem using the DirichletNeumann method of Section 4.1. We had the method iterate until the relative size of the correction in $p$ measured in the energy norm dropped below $10^{-13}$. This is shortly before rounding errors prevent further progress. The subdomain problems were solved in the generalized pressure $u_{i}, i=1,2$, by uniform multigrid iterating until the relative norm of the correction dropped below $10^{-12}$.

We first consider the error of the multidomain discretization (32)-(34) in the generalized pressure $u$. For this note that the function $u: \Omega \rightarrow \mathbb{R}$ with $u_{\mid \Omega_{i}}=u_{i}$ defined by

$$
u_{i}:=\kappa_{i}\left(p_{i}\right) \quad \text { in } \Omega_{i}
$$

is discontinuous across $\Gamma$ and solves the generalized multidomain problem (18)- 200 with the same source term $f$ as given in (55). We compute discrete solutions $u_{l, i}$, $i=1,2$, and measure the error against the analytical solution $u$ in the $L^{2}$-norm on $\Omega$ and, since $u$ is discontinuous across $\Gamma$, in the broken $H^{1}$-norm on $\Omega_{1} \cup \Omega_{2}$. For the latter, the gradient of $u$ is needed on the subdomains. Using the chain rule (7), i.e., $\nabla u_{i}=k r_{i}\left(\theta_{i}\left(\tilde{p}_{i}\right)\right) \nabla \tilde{p}_{i}$, which is valid for $p_{i} \in H^{1}\left(\Omega_{i}\right)$, see [27], we can reuse the formulas for $p_{i}$ and apply a quadrature rule to compute the integrals. We use the composite quadrature rule with three steps of refinement and a fifth-order rule on each subelement. The result can be seen in Figure 7 left. Both the $L^{2}$ and the $H^{1}$-error of the discrete generalized multidomain formulation (32)-(34) show optimal convergence orders $h^{2}$ and $h$, respectively, but only after about $5-7$ refinement steps. The reason is that the source terms $f_{i}$ contain discontinuities and steep gradients that can only be resolved on sufficiently fine grids.

Now $p_{l} \in \mathcal{S}_{l}$ is obtained from $u_{l, i}, i=1,2$ by inverse discrete Kirchhoff transformation (37). We computed the error $\left\|p-p_{l}\right\|$ in the $L^{2}$-norm and in the $H^{1}$-norm. The result can be seen in Figure 7 right. Again, optimal convergence orders are obtained once the grid is sufficiently fine to resolve the features of the right hand

1 www.dune-project.org 

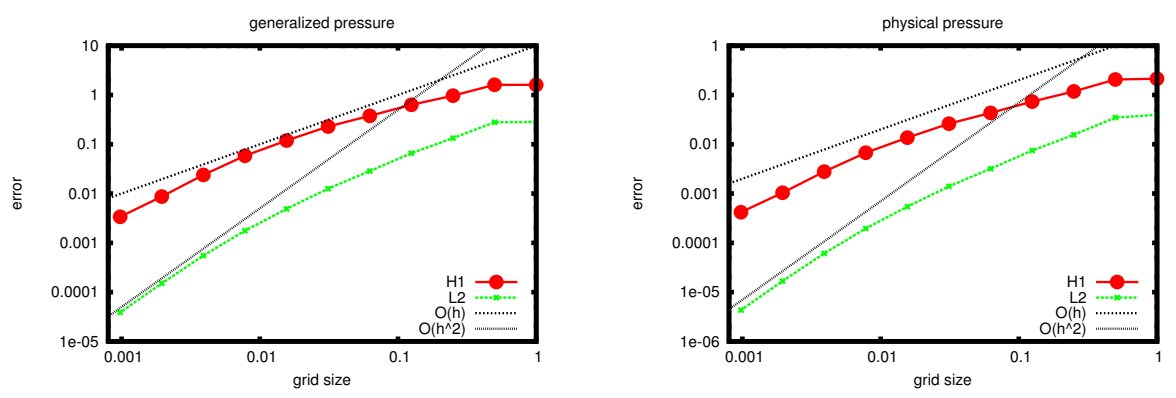

Figure 7. Discretization errors as a function of grid size $h$. Left: generalized pressure $u$. Right: physical pressure $p$

side $f$. In fact, the error reduction curves for $p$ and for $u$ look quite similar in the graphics and seem to differ just by approximately the factor 10, whose inverse is about the order of magnitude of the bubbling pressures in Table1. This reflects the fact that in our concrete calculations we scale $u$ by the bubbling pressure to obtain an adimensional variable. Thus the inverse Kirchhoff transformations $\kappa_{i}^{-1}: \mathbb{R} \rightarrow \mathbb{R}$, $i=1,2$, seem to have hardly an influence on the discretization error in this case, which suggests that their mean Lipschitz constants on the images of $u_{i}$ are of order of magnitude 1. In particular, we do not observe saturation of $L^{2}$ - or increase of $H^{1}$ discretization errors for the physical pressure $p$ here in contrast to the experimental discretization result reported in [13, Sec. 5.1]. The reason is that the potentially ill-conditioned inverse discrete Kirchhoff transformations amplify round-off errors at most by a factor of $\max _{x \in \Omega_{i}, i=1,2}\left(\kappa_{i}^{-1}\right)^{\prime}\left(u_{i}(x)\right) \approx 39$.

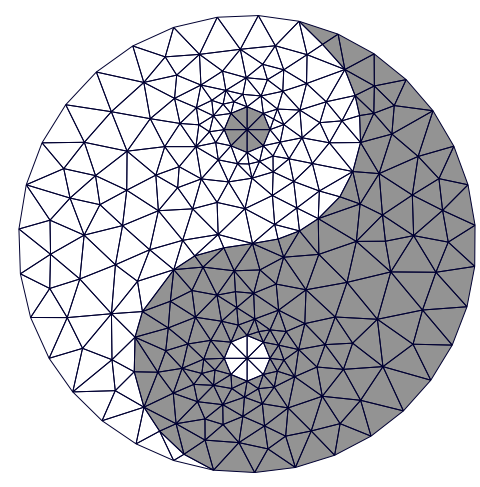

Figure 8. Yin-Yang domain and grid

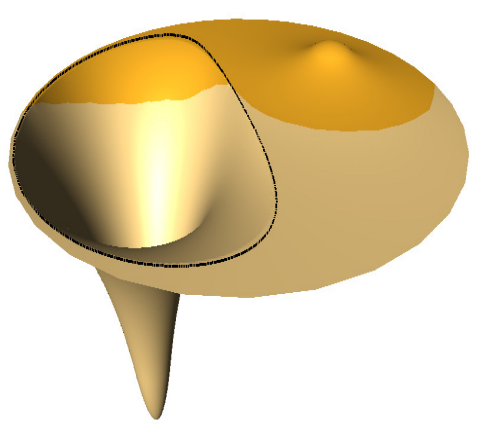

Figure 9. Typical shape of solution with saturation front

\subsection{Robustness of substructuring with respect to Brooks-Corey parame-} ters. We consider the multidomain formulation 14 -16$)$ for the Richards equation on the Yin-Yang domain $\Omega$ with radius one, as depicted in Figure 8. As in the previous subsection we omit the gravity term, but now we incluce source terms, i.e., we have $f_{i}=\bar{f}_{i}+n_{i} \theta_{i}\left(\bar{p}_{i}\right)$ and $f_{i j}=f_{j i}=0$. The white subdomain together with the grey circle $B_{1}$ and the grey subdomain with the white circle $B_{2}$ are denoted by $\Omega_{1}$ 
and $\Omega_{2}$, respectively. We select $\left.\bar{f}_{1}\right|_{B_{1}}=5 \cdot 10^{-5} \mathrm{~s}^{-1},\left.\bar{f}_{2}\right|_{B_{2}}=-10^{-1} \mathrm{~s}^{-1}$, and zero elsewhere, so that $B_{1}$ and $B_{2}$ can be regarded as a source and a sink, respectively. The reference soil parameters corresponding to sand are given in Table 1 , and are chosen to be the same in both domains. The problem is discretized as described in Section 3 with the time step size $\tau_{1}=1 s$ and a spatial mesh $\mathcal{T}_{4}$ resulting from four uniform refinements of the initial triangulation $\mathcal{T}_{0}$ depicted in Figure 8 . We consider the algebraic solution of the spatial problem occurring in the first time step, starting from the initial condition $p_{0}(x)=0.45(|x|-1)$.

In contrast to homogeneous Brooks-Corey parameters, ill-conditioning and algebraic solution are no longer separated by the discretization in case of different soil parameters $\lambda_{i}$ or $p_{b, i}$ on both sides of $\Gamma$, because the evaluation of the inverse Kirchhoff transformation appears in the transmission conditions (15). Nevertheless, in [12] we have presented nontrivial examples for which the nonlinear versions of both the Dirichlet-Neumann and the Robin methods (45)-(48) and (52)-(54) ) show similar mesh dependence as their counterparts in the linear self-adjoint case. Here we concentrate on robustness of these methods with respect to jumps in the pore size distributions $\lambda_{i}$ and the bubbling pressures $p_{b, i}$ in $\Omega_{i}, i=1,2$. To this end, we consider variations

$$
10^{-2} \leq \lambda_{2} \leq 1, \quad 10^{-2} \leq-p_{b, 2} \leq 1
$$

in $\Omega_{2}$, the domain with the sink. The ranges are chosen to cover all physically relevant parameters, compare [32, Table 5.3.2]. In $\Omega_{1}$ we always keep the reference values $\lambda_{1}$ and $p_{b, 1}$ of sand. See Figure 9 for how a solution typically looks like in these cases.

Convergence rate measurements and termination criteria are implemented using the subdomain energy norms given by

$$
\left\|v_{i}\right\|_{i}^{2}=a_{i}\left(v_{i}, v_{i}\right) \quad \forall v_{i} \in V_{i} \quad i=1,2,
$$

on the spaces defined in 21. The local problems are solved by the monotone multigrid method of [13] until for the iterates $v_{i}^{j}, i=1,2, j=1,2, \ldots$, the relative norm of the correction $\left\|v_{i}^{j+1}-v_{i}^{j}\right\|_{i} /\left\|v_{i}^{j}\right\|_{i}$ dropped below $10^{-12}$. For convergence rate estimates of the substructuring algorithms we used the 2-norm on $V_{1} \oplus V_{2}$ defined by

$$
\|v\|=\left(\left\|v_{1}\right\|_{1}^{2}+\left\|v_{2}\right\|_{2}^{2}\right)^{1 / 2} \quad \forall v=\left(v_{1}, v_{2}\right) \in V_{1} \oplus V_{2} .
$$

The convergence rate of the substructuring iteration with the iterates $u^{\nu} \in$ $\mathcal{S}_{4,1} \otimes \mathcal{S}_{4,2}, \nu=1,2, \ldots$, in the generalized pressure is approximated by taking the arithmetic mean of the geometric means

$$
\rho_{\nu}=\sqrt[\nu]{\frac{\left\|u^{\nu+1}-u^{\nu}\right\|}{\left\|u^{1}-u^{0}\right\|}}
$$

for $\nu=\left\lfloor\nu_{0} / 3\right\rfloor, \ldots, \nu_{0}$ with $\nu_{0}$ chosen such that $\left\|u^{\nu_{0}+1}-u^{\nu_{0}}\right\| \leq 10^{-8}$. The initial iterate is chosen as the Lagrange interpolated discrete Kirchhoff-transformed initial condition $p_{0}$.

5.2.1. Dirichlet-Neumann method. We first consider the Dirichlet-Neumann iteration as described in Subsection 4.1. A Dirichlet problem on $\Omega_{1}$ and a corresponding Neumann problem on $\Omega_{2}$ is solved in each iteration step. For each set of parameters, an approximation of the optimal damping parameter $\vartheta$ is determined by a 

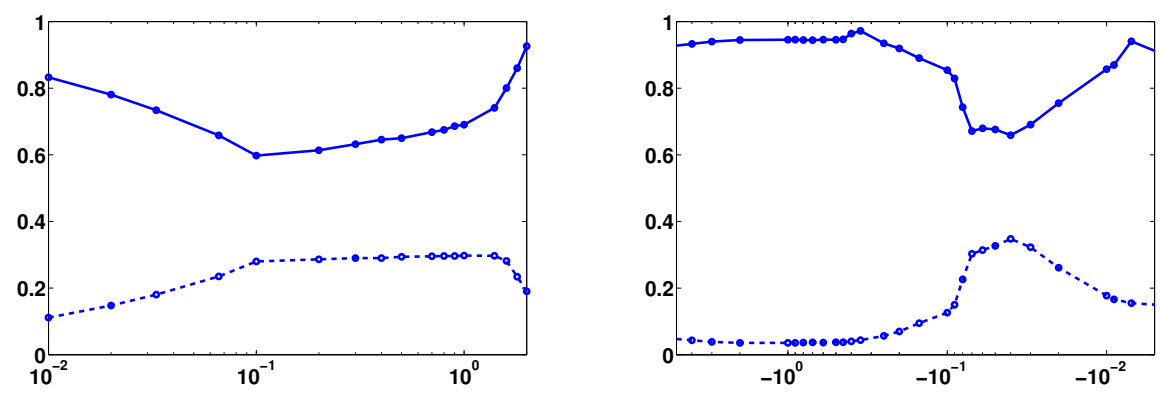

Figure 10. Convergence rates $\rho_{\nu}$ (solid line) and damping factor $\vartheta$ (dashed line) of the Dirichlet-Neumann method over pore size distribution factor $\lambda_{2}$ (left) and bubbling pressure $p_{b, 2}$ (right) in subdomain $\Omega_{2}$

heuristic strategy. Concretely, we applied the method first for damping parameters coming from a coarse sampling of the parameter interval $[0,1]$ and then from a finer sampling of the region where good convergence rates have been observed.

The left picture of Figure 10 shows the convergence rate $\rho$ as a function of the pore size distribution $\lambda_{2}$ in $\Omega_{2}$ (solid line) together with the associated damping parameter $\vartheta$ (dashed line). It turns out that the method convergences for the whole range of $\lambda_{2}$. While $\rho \leq 0.75$ holds for $10^{-1} \leq \lambda_{2} \leq 1$, convergence rates deteriorate outside of this interval.

The right picture of Figure 10 indicates that the convergence speed of the Dirichlet-Neumann method is more sensitive to variations of the bubbling pressure $p_{b, 2}$ in $\Omega_{2}$. Outside of a window of $3 \cdot 10^{-2} \leq-p_{b, 2} \leq 7 \cdot 10^{-2}$, where $\rho<0.7$ is reached, the convergence rate quickly deteriorates up to values of $\rho>0.9$ (solid line). Interestingly, in both pictures, $\rho$ and $\vartheta$ seem to always add up to one. This mirrors a known fact from the linear theory which can be easily verified in 1D, see, e.g., [31, p. 12].

5.2.2. Robin method. For a comparison we now consider the Robin method presented in Subsection 4.2 where we prescribe the acceleration parameter $\gamma=\gamma_{1}=\gamma_{2}$. Similarly as for the Dirichlet-Neumann method we determine an approximation of the optimal $\gamma$ by a heuristic strategy based on coarse sampling of the relevant parameter interval followed by a finer sampling of regions exhibiting good convergence rates.

The dependence of the convergence rate $\rho$ of the Robin iteration on the pore size distribution factor $\lambda_{2}$ is depicted in the left picture of Figure 11. Corresponding optimal acceleration parameters $\gamma$ are in the interval $\left[10^{-3}, 10^{-2}\right]$ and generally tend to be bigger the smaller $\rho$ is.

It turns out that $\rho<0.5$ holds throughout the whole range of $\lambda_{2}$. Moreover, the right picture in Figure 11 indicates a similar robustness with respect to variation of the bubbling pressure $p_{b, 2}$ for a similar range of parameters $\gamma \approx 0.5 \cdot 10^{-2}$. Theoretical justification of these promising results will be subject of future research. Since the Robin method shows a better behaviour than the Dirichlet-Neumann method in these parameter studies we apply the Robin method in the hydrological example to which we turn now. 

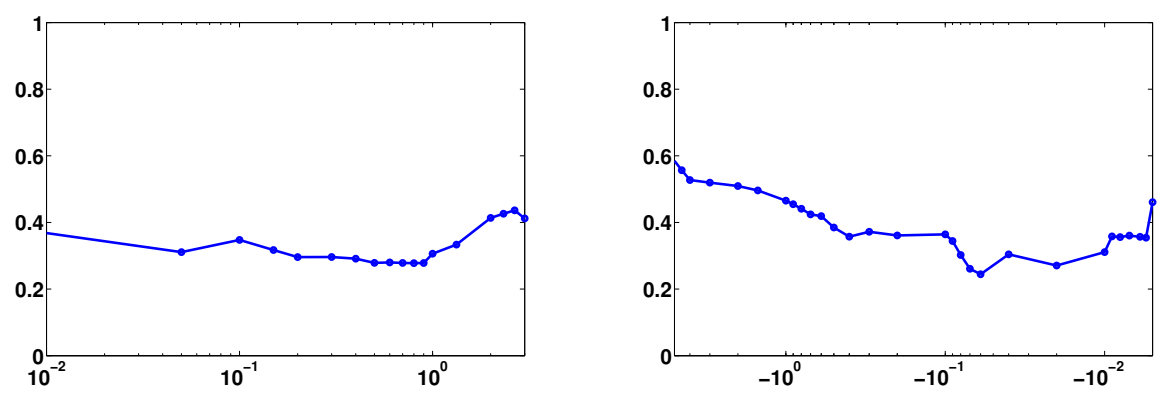

FiguRe 11. Convergence rates $\rho_{\nu}$ of the Robin method over pore size distribution factor $\lambda_{2}$ (left) and bubbling pressure $p_{b, 2}$ (right) in subdomain $\Omega_{2}$

5.3. Seepage of ground and surface water. The final numerical example contains a hydrologically realistic setting with four different soil layers and including surface water. We first describe the surface water model and its coupling to the Richards equation before giving the details and results of the concrete example. For an exhaustive exposition of the example see [8, Sec. 4.3].

5.3.1. Coupling of the Richards equation with surface water: a compartment model. The principle of the reservoir model as depicted in Figure 12 is the following. First, we assume that the surface water is non-moving and that for (almost) all times $t>0$, the water table is horizontal and uniquely determined by a real number $h(t)$, the height of water with respect to the lowest point of the lake. If the geometry that surrounds the lake is given, knowing $h(t)$ is equivalent to knowing the volume $V(h(t))$ and the mass $m(h(t))$ of surface water in the reservoir.

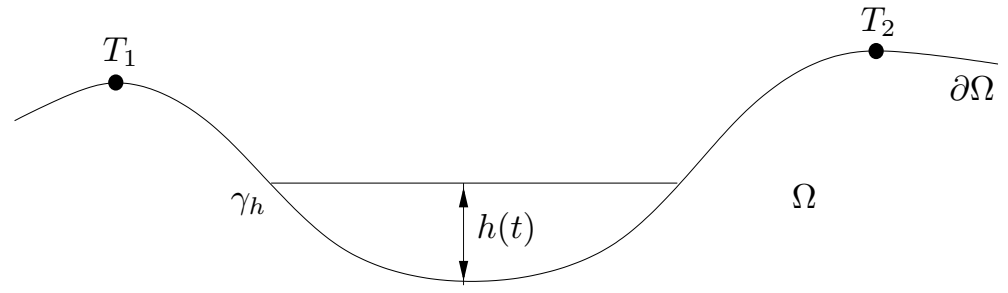

Figure 12. Flow across $\gamma_{h}$ between $T_{1}$ and $T_{2}$ affects lake height $h(t)$

The continuous case. We assume that by its hydrostatic pressure, the height $h(t)$ of surface water imposes a Dirichlet boundary condition $\tilde{p}_{D}=h(t)$ for the Richards equation (recall that the physical pressure in (1) is already measured in meters of a water column). On the other hand we assume mass conservation, i.e., the flow of water out of or into the ground affects the height or volume or mass of the lake corresponding to its mass gain or loss, respectively. With the water density $\varrho$ this condition on the normal flux $\tilde{\mathbf{v}} \cdot \mathbf{n}$ out of $\Omega$ can be formulated as

$$
\frac{d}{d t} m(h(t))=\varrho \frac{d}{d t} V(h(t))=\varrho \int_{\gamma_{h}} \tilde{\mathbf{v}}(x, t) \cdot \mathbf{n} d \sigma(x) .
$$


Here, $\gamma_{h}$ is the portion of $\partial \Omega$ between the top points $T_{1}$ and $T_{2}$ in Figure 12, on which the flow of water into or out of the ground affects the lake height. Altogether, the compartment model with the mass conservation (56) and the Dirichlet condition $\tilde{p}_{D}=h(t)$ as coupling conditions result in the coupling of an ordinary and a partial differential equation. For another example of that kind we refer to [15].

The time-discrete case. While we use an implicit-explicit discretization for the Richards equation, we choose an explicit one for the surface water, i.e. for the ODE (56). Concretely, the time-discrete volume $V$ of the lake at the time $t_{k}$ is

$$
V:=\bar{V}+\int_{\gamma_{h}} \overline{\mathbf{v}}(x) \cdot \mathbf{n} d \sigma(x),
$$

where $\bar{V}$ is the time-discrete volume of the lake at the time $t_{k-1}$ and $\overline{\mathbf{v}}$ is the timediscrete water flux for the time $t_{k-1}$. The time-discrete solution for the Richards equation at the time $t_{k}$ is then given by the lake height that corresponds to $V$ and serves as the Dirichlet boundary value $p_{D}$.

An implicit time discretization of (56) would require an additional solution technique for the coupled PDE and ODE. See [5, 16] for iteration methods of DirichletNeumann- or Robin-Neumann-type applied to coupling problems of this kind.

A weak formulation of (57) can only be given approximately, since

$$
\int_{\gamma_{h}} \overline{\mathbf{v}}(x) \cdot \mathbf{n} d \sigma(x)=\int_{\partial \Omega} \overline{\mathbf{v}}(x) \cdot \mathbf{n} \cdot \mu d \sigma(x)
$$

might only hold for $\mu$ on $\partial \Omega$ with $\mu=1$ on $\gamma_{h}$ and $\mu=0$ on $\partial \Omega \backslash \gamma_{h}$. This, however, does not define an element of $H^{1 / 2}(\partial \Omega)$ so that we cannot apply an extension operator to $\mu$ (as in (30) ) in order to obtain a weak form of the right hand side of (58) by Green's formula. Since this problem occurs in the fully discrete case, too, we need to approximate $\mu$ by a suitable element of $H^{1 / 2}(\partial \Omega)$.

The fully discrete case. For the finite element discretization of the time-discretized mass conservation (57) we approximate the integral as follows. In view of Figure 12 , we consider the set $\mathcal{N}_{h}$ of all vertices of the grid lying on $\gamma_{h}$ between $T_{1}$ and $T_{2}$ (or possibly equal to these points). We define the finite element function $\mu_{h}$ on $\partial \Omega$ that is 1 in each of the nodes in $\mathcal{N}_{h}$ and 0 in all other vertices of $\partial \Omega$. The finite element function $v_{h}$ shall be its trivial extension on $\Omega$ given by $v_{h}(q)=0$ for all $q \in \mathcal{N}$. The discretization of (57) is motivated by applying Green's formula to the right hand side of $(58)$ with $\mu_{h}$ instead of $\mu$ and discretizing the obtained weak form just as (34) was obtained from (30). Therefore, with the discrete volume $\bar{V}_{l}$ from the former time step we define the new discrete volume by

$$
V_{l}:=\bar{V}_{l}-\sum_{q \in \mathcal{N}_{h}} M_{1}\left(u_{l, 1}(q)\right) \mu_{h}(q) h_{q, 1}-a_{1}\left(u_{l, 1}, v_{h}\right)+\ell_{l, 1}\left(v_{h}\right),
$$

where $u_{l, 1}$ is the solution of $(32)-34$ for the top subdomain at the time $t_{k-1}$.

For simplicity we assume that the geometry of the lake can be approximated by a circle line. Then, in order to determine the height $h_{l}$ of the lake approximatively from the obtained volume $V_{l}$ at the time $t_{k}$ we use numerical integration of the circle segment representing $V_{l}$. 


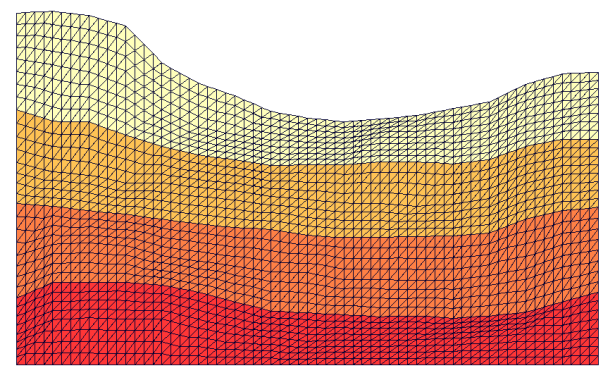

Figure 13. Fine grid, four soil layers as in Table 2

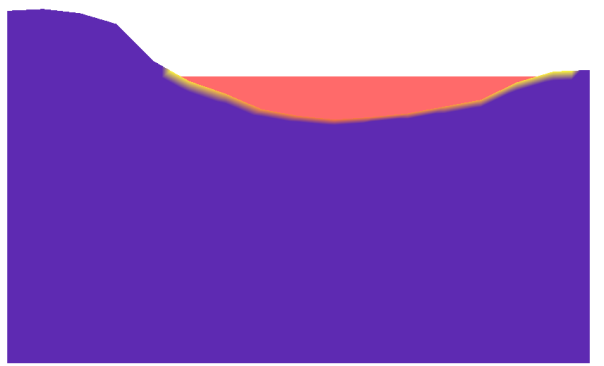

Figure 14. Initial condition: dry soil and surface water

5.3.2. Setting and numerical results. With regard to our concrete example, Figure 13 shows the domain $\Omega \subset \mathbb{R}^{2}$ decomposed into the four subdomains $\Omega_{1}, \Omega_{2}$, $\Omega_{3}$ and $\Omega_{4}$ from the top to the bottom. The width of the domain is $2 \mathrm{~m}$, and the height from the bottom to the highest point of $\Omega$ is approximately $1.214 \mathrm{~m}$. We apply the Brooks-Corey parameter functions (2) and (3). We choose the soil parameters of sand, loamy sand, sandy loam and loam as given in Table 2 (compare [32, Tables 5.3.2 and 5.5.5]). Figure 13 shows the finest grid (with the mesh size $h=0.038$ ) which we obtain on the third refinement level with 585 nodes in each subdomain.

\begin{tabular}{|c||c|c|c|c|c|c|}
\hline$\Omega_{i}$ & $n_{i}$ & $\theta_{m, i}$ & $\theta_{M, i}$ & $\lambda_{i}$ & $p_{b, i}[\mathrm{~m}]$ & $K_{h, i}[\mathrm{~m} / \mathrm{s}]$ \\
\hline \hline$i=1$ (sand) & 0.437 & 0.0458 & 1 & 0.694 & -0.0726 & $6.54 \cdot 10^{-5}$ \\
\hline$i=2$ (loamy sand) & 0.437 & 0.0801 & 1 & 0.553 & -0.0869 & $1.66 \cdot 10^{-5}$ \\
\hline$i=3$ (sandy loam) & 0.453 & 0.0905 & 1 & 0.378 & -0.1466 & $6.06 \cdot 10^{-6}$ \\
\hline$i=4$ (loam) & 0.463 & 0.0583 & 1 & 0.252 & -0.1115 & $3.67 \cdot 10^{-6}$ \\
\hline
\end{tabular}

TABLE 2. Soil parameters in the four layers from top to bottom

As the initial condition depicted in Figure 14 in a colourplot we choose $p=-10$ (meters of a water column) corresponding to a practically dry soil in $\Omega$ except for the nodes on the top boundary which are covered by surface water (red in Figure 14), where a hydrostatic pressure from the lake is given. The height of the lake at the time $t=0 \mathrm{~s}$ is $0.1686 \mathrm{~m}$, the radius of the circle line by which we approximate $\gamma_{h}$ is $r=1.2 \mathrm{~m}$.

The hydrological situation on the banks of the lake is described by Signorini-type boundary conditions on the rest of the top boundary of $\Omega$, see Subsection 2.2 . In the time evolution we impose a constant inflow of $-\mathbf{v} \cdot \mathbf{n}=3 \cdot 10^{-4} \mathrm{~m} / \mathrm{s}$ across the lower half of the left boundary of $\Omega_{1}$. On the rest of the left boundary of $\Omega$ as well as on the right and the bottom part of $\partial \Omega$ homogeneous Neumann boundary conditions $\mathbf{v} \cdot \mathbf{n}=0$ are assumed for all time steps. For the time evolution we choose the constant time step size $\tau=10 \mathrm{~s}$. 
Based on the numerical results in Subsection 5.2 we choose the Robin method (52)-54 for the solution of the spatial problems. We use the acceleration parameter $\gamma=10^{-4}$ suggested by numerical experiments for all subdomains and at all time steps. In addition, note that the Dirichlet-Neumann method would encounter nonuniqueness issues with the Neumann data imposed on $\partial \Omega$. As in Subsections 5.1 and 5.2 , the inner solver we applied to the homogeneous problem on each subdomain $\Omega_{i}$ for $i=1,2,3,4$, is the monotone multigrid method. We stopped this iteration when the relative size of the corrections of $u_{i}$ in the energy norm dropped below $10^{-12}$.

In each time step we determine the discrete physical pressure $p$ in the finite element space $\mathcal{S}$, which is given by the mesh and the boundary conditions, up to an accuracy of $1 \%$ in the (semi-)norm

$$
\|p\|:=\left(\sum_{i=1}^{4} a_{i}\left(p_{i}, p_{i}\right)\right)^{1 / 2}, \quad p \in \mathcal{S}, \quad p_{i}:=p_{\mid \Omega_{i}}, \quad i=1,2,3,4 .
$$

Intensive numerical tests show that we may assume a maximal convergence rate of $\rho=0.95$ for the Robin iteration in the whole evolution. Assuming $\|p\| \approx\left\|p^{k}\right\|$ for the Robin iterates $p^{k}, k \geq 0$, is justified since the initial iterate is the solution of the previous time step and the time step size $\tau=10 \mathrm{~s}$ is moderate. Therefore, the stopping criterion

$$
\frac{\left\|p^{k+1}-p^{k}\right\|}{\left\|p^{k}\right\|}<0.0005
$$

gives the desired accuracy

$$
\frac{\left\|p-p^{k}\right\|}{\|p\|}<\frac{1}{1-0.95} \cdot 0.0005=0.01
$$

Figure 19 shows the number of Robin iterations needed per time step with this stopping criterion for the evolution displayed in Figures 15, 18. Mostly we obtain iteration numbers below 15 except for the first few time steps, in which the evolution is considerably faster as the saturation front crosses the first layer of sand with a relatively big hydraulic conductivity $K_{h, 1}$. Here the solution from the previous time step as the initial iterate for the next time step is further away from the next solution than later in the evolution when the saturation front is slower as it crosses layers with smaller hydraulic conductivity. As Figures 15, 18 show, the evolution contains emptying of the lake, a dry lake and again rising of the water level until at the end a fully saturated soil is reached for which the boundary value problems for the Richards equation become linear Darcy problems. The range of $p$ varies from -10 (blue in the colour plots) over $p \approx 0$ in the yellow regions to $p \approx 1$ (orange) until $p \approx 2$ in the red regions.

Recall from Subsection 3.2 .2 that we use a time-explicit upwind discretization of the convective (gravitational) term. We do not see instabilities in the numerical solutions due to this term. Our time step size $\tau=10$ s obeys the CFL condition for linear cases which requires

$$
\tau<\frac{n_{1}}{K_{h, 1}\left(3+\frac{2}{\lambda_{1}}\right)} h=43.2 \mathrm{~s}
$$

with our data, compare [8, Sec. 4.2.1]. 


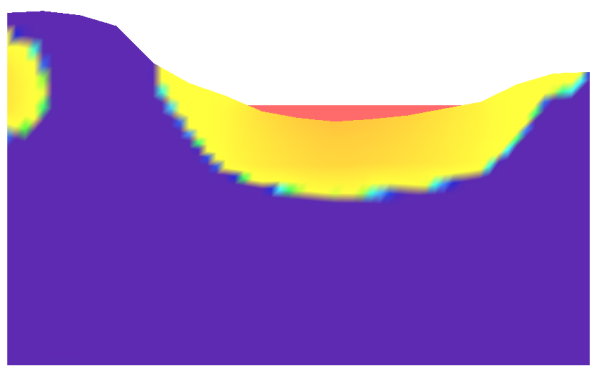

FiguRE 15. $t=200$

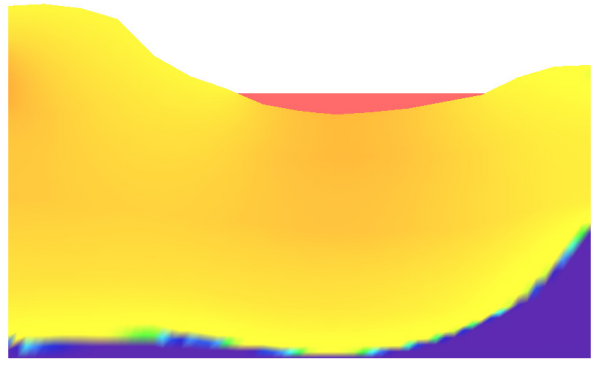

FigURE 17. $t=10000$

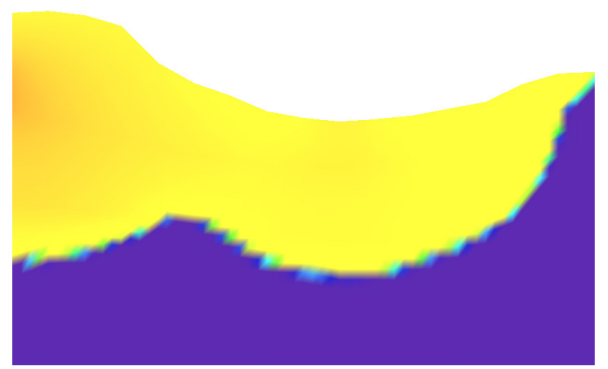

FiguRe 16. $t=4000$

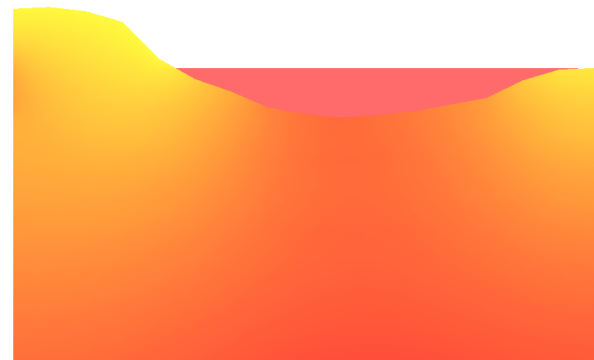

FiguRE 18. $t=13420$

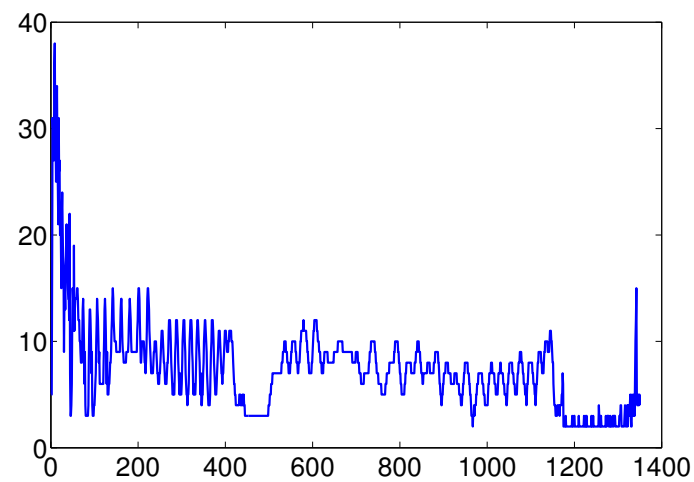

FIgURE 19. Number of iterations per time step of the Robin method with stopping criterion (59) for a relative accuracy 60 of $1 \%$

Altogether, the nature of our example and the numerical results we obtained demonstrate that the solution method we propose for the Richards equation in heterogeneous layered soil with surface water can be successfully applied to a realistic hydrological model problem. 


\section{ACKNOWLEDGEMENTS}

The authors would like to thank Jan Schreiber for his assistance in the production of the parameter studies.

\section{REFERENCES}

[1] H.W. Alt and E. DiBenedetto. Nonsteady flow of water and oil through inhomogeneous porous media. Ann. Scuola Norm. Sup. Pisa Cl. Sci., 12:335-392, 1985.

[2] H.W. Alt and S. Luckhaus. Quasilinear elliptic-parabolic differential equations. Math. Z., 183:311-341, 1983.

[3] H.W. Alt, S. Luckhaus, and A. Visintin. On nonstationary flow through porous media. Ann. Math. Pura Appl., 136:303-316, 1984.

[4] P. Bastian, O. Ippisch, F. Rezanezhad, H.J. Vogel, and K. Roth. Numerical simulation and experimental studies of unsaturated water flow in heterogeneous systems. In W. Jäger, R. Rannacher, and J. Warnatz, editors, Reactive Flows, Diffusion and Transport, pages 579-598. Springer, 2005.

[5] P. Bastian, M. Blatt, A. Dedner, C. Engwer, R. Klöfkorn, R. Kornhuber, M. Ohlberger, and O. Sander. A generic interface for adaptive and parallel scientific computing. Part II: implementation and tests in DUNE. Computing, 82(2-3):121-138, 2008.

[6] P. Bastian, G. Buse, and O. Sander. Infrastructure for the coupling of Dune grids. In Proc. ENUMATH 2009, pages 107-114. Springer, 2010.

[7] J. Bear. Dynamics of Fluids in Porous Media. Dover Publications, 1988.

[8] H. Berninger. Domain Decomposition Methods for Elliptic Problems with Jumping Nonlinearities and Application to the Richards Equation. PhD thesis, Freie Universität Berlin, 2007.

[9] H. Berninger. Non-overlapping domain decomposition for the Richards equation via superposition operators. In M. Bercovier, M.J. Gander, R. Kornhuber, and O. Widlund, editors, Domain Decomposition Methods in Science and Engineering XVIII, volume 70 of LNCSE, pages 169-176. Springer, 2009.

[10] H. Berninger and O. Sander. Substructuring of a Signorini-type problem and Robin's method for the Richards equation in heterogeneous soil. Comput. Vis. Sci., 13(5):187-205, 2010.

[11] H. Berninger, R. Kornhuber, and O. Sander. On nonlinear Dirichlet-Neumann algorithms for jumping nonlinearities. In O.B. Widlund and D.E. Keyes, editors, Domain Decomposition Methods in Science and Engineering XVI, volume 55 of LNCSE, pages 483-490. Springer, 2007.

[12] H. Berninger, R. Kornhuber, and O. Sander. Convergence behaviour of Dirichlet-Neumann and Robin methods for a nonlinear transmission problem. In Y. Huang, R. Kornhuber, O. Widlund, and J. Xu, editors, Domain Decomposition Methods in Science and Engineering XIX, volume 78 of LNCSE, pages 87-98. Springer, 2010.

[13] H. Berninger, R. Kornhuber, and O. Sander. Fast and robust numerical solution of the Richards equation in homogeneous soil. SIAM J. Numer. Anal., 49 (6):2576-2597, 2011. 
[14] H. Berninger, S. Loisel, and O. Sander. The 2-Lagrange Multiplier Method Method Applied to Nonlinear Transmission Problems for the Richards Equation in Heterogeneous Soil with Cross Points. Submitted to SIAM J. Sci. Comp., 2012. IGPM Preprint 349, RWTH Aachen University.

[15] H. Berninger, M. Ohlberger, O. Sander, and K. Smetana. Unsaturated subsurface flow with surface water and nonlinear in- and outflow conditions. 2012. Submitted to $M 3 A S$ and deposited on http://arxiv.org/abs/1301.2488v1.

[16] H. Berninger, R. Kornhuber, and O. Sander. Heterogeneous substructuring methods for coupled surface and subsurface flow. In Domain Decomposition Methods in Science and Engineering XX, LNCSE. Springer, 2013. to appear.

[17] F. Brezzi and G. Gilardi. Functional spaces. In H. Kardestuncer and D.H. Norrie, editors, Finite Element Handbook, chapter 2 (part 1), pages 1.29-1.75. Springer, 1987.

[18] R.J. Brooks and A.T. Corey. Hydraulic properties of porous media. Technical Report Hydrology Paper No. 3, Colorado State University, Civil Engineering Department, Fort Collins, 1964.

[19] N.T. Burdine. Relative permeability calculations from pore-size distribution data. Petr. Trans., Am. Inst. Mining Metall. Eng., 198:71-77, 1953.

[20] G. Chavent and J. Jaffré. Dynamics of Fluids in Porous Media. Elsevier Science, 1986.

[21] H. Darcy. Les Fontaines Publiques de la Ville de Dijon. Appendice. - Note D. Dalmont, Paris, 1856.

[22] I. Ekeland and R. Temam. Convex Analysis and Variational Problems. NorthHolland, 1976.

[23] R. Eymard, M. Gutnic, and D. Hilhorst. The finite volume method for Richards equation. Comput. Geosci., 3(3-4):259-294, 1999.

[24] J. Fuhrmann. On numerical solution methods for nonlinear parabolic problems. In R. Helmig, W. Jäger, W. Kinzelbach, P. Knabner, and G. Wittum, editors, Modeling and Computation in Environmental Sciences, First GAMM-Seminar at ICA Stuttgart, pages 170-180. Vieweg, 1997.

[25] M.J. Gander. Optimized Schwarz methods. SIAM J. Numer. Anal., 44(2): 699-731, 2006.

[26] D. Kröner. Numerical Schemes for Conservation Laws. Wiley-Teubner, 1997.

[27] G. Leoni and M. Morini. Necessary and sufficient conditions for the chain rule in $W_{\text {loc }}^{1,1}\left(\mathbb{R}^{N} ; \mathbb{R}^{d}\right)$ and $B V_{\text {loc }}\left(\mathbb{R}^{N} ; \mathbb{R}^{d}\right)$. J. Eur. Math. Soc. (JEMS), 9(2): 219-252, 2007.

[28] S.H. Lui. A Lions non-overlapping domain decomposition method for domains with an arbitrary interface. IMA J. Numer. Anal., 29(2):332-349, 2009.

[29] M. Marcus and V.J. Mizel. Complete characterization of functions which act, via superposition, on Sobolev spaces. Trans. Amer. Math. Soc., 251:187-218, 1979 .

[30] I.S. Pop and B. Schweizer. Regularization schemes for degenerate Richards equations and outflow conditions. Math. Models Methods Appl. Sci, 21:1685$1712,2011$.

[31] A. Quarteroni and A. Valli. Domain Decomposition Methods for Partial Differential Equations. Oxford Science Publications, 1999.

[32] W.J. Rawls, L.R. Ahuja, D.L. Brakensiek, and A. Shirmohammadi. Infiltration and soil water movement. In D.R. Maidment, editor, Handbook of Hydrology, 
chapter 5. McGraw-Hill, 1993.

[33] L.A. Richards. Capillary conduction of liquids through porous mediums. Physics, 1:318-333, 1931.

[34] Oliver Sander. Geodesic finite elements in spaces of zero curvature. In A. Cangiani, R.L. Davidchack, E.H. Georgoulis, A. Gorban, J. Levesley, and M.V. Tretyakov, editors, Proc. of Enumath, 2011.

[35] E. Schneid, P. Knabner, and F. Radu. A priori error estimates for a mixed finite element discretization of the Richards' equation. Numer. Math., 98(2): 353-370, 2004.

[36] B. Schweizer. Regularization of outflow problems in unsaturated porous media with dry regions. J. Differ. Equations, 237(2):278-306, 2007.

[37] C.A. San Soucie. Mixed finite element methods for variably saturated subsurface flow. PhD thesis, Rice University, 1996.

[38] C. Wagner, G. Wittum, R. Fritsche, and H.-P. Haar. Diffusions-Reaktionsprobleme in ungesättigten porösen Medien. In K.-H. Hoffmann, W. Jäger, T. Lohmann, and H. Schunck, editors, Mathematik: Schlüsseltechnologie für die Zukunft. Verbundprojekte zwischen Universität und Industrie, pages 243253. Springer, 1997. 